\title{
Asymptotic normality of the relative error regression function estimator for censored and time series data
}

https://doi.org/10.1515/demo-2021-0107

Received October 9, 2020; accepted July 18, 2021

Abstract: Consider a survival time study, where a sequence of possibly censored failure times is observed with d-dimensional covariate The main goal of this article is to establish the asymptotic normality of the kernel estimator of the relative error regression function when the data exhibit some kind of dependency. The asymptotic variance is explicitly given. Some simulations are drawn to lend further support to our theoretical result and illustrate the good accuracy of the studied method. Furthermore, a real data example is treated to show the good quality of the prediction and that the true data are well inside in the confidence intervals.

Keywords: Asymptotic normality, censored data, kernel smoothing, probability consistency, regression function, relative error, strong mixing.

MSC: 62G05, 62G08, 62G30, 62N01, 62N02, 62P10

\section{Introduction}

Studying the relationship between two random variables is a very important issue in statistics. Let $(T, X)$ be a random vector where $T$ represents the response variable and $X$ is a $d$-dimensional covariates. We assume that $T$ is subject to random right censoring, i.e. instead of observing $T$ we only observe the couple $(Y, \delta)$, where $Y=\min (T, C), \delta=\mathbb{1}_{\{T \leq C\}}$ where $\mathbb{1}_{A}$ denotes the indicator function of the set $A$ and $C$ represents the censoring time. The importance of studying this kind of data is highlighted by the application of the latter to real data, see among other references [1] and [22].

In the statistical literature it is very common to assume that $(T, X)$ and $C$ are independent. Under this assumption a lot of work has been done on the nonparametric estimation of the regression operator issue from $T=m(X)+\varepsilon$ where $\varepsilon$ is the observation error term. For a review about nonparametric regression using a local linear technical, we refer the reader to [11].

However, in several practical situations it is not realistic to assume that the data are independent. Consider for example the situation where the patients from the same hospital have correlated survival times due to unmeasured variables like the quality of the hospital equipment. An example of such data can be found in [21]. In economic duration data, event times are often correlated and the observation of the event may be prevented by the occurrence of an earlier competing event called censoring. Another example is the observations on duration of unemployment, which may be right censored and are typically correlated. For real data, the reader can refer to [29], [4] in such a case the data are correlated. This was the reason that motivated us to consider in this paper the situation when the data exhibit some kind of dependency.

Feriel Bouhadjera: Université Badji-Mokhtar, Lab. de Probabilités et Statistique. BP 12, 23000 Annaba, Algérie. Université du Littoral Côte d'Opale. Lab. de Math. Pures et Appliquées. 50 Rue Ferdinand Buisson, 62100, Calais, France. E-mail: feriel.bouhadjera@univ-lille.fr

*Corresponding Author: Elias Ould Saïd: Université du Littoral Côte d’Opale. Lab. de Math. Pures et Appliquées. IUT de Calais. 19, rue Louis David. 62228, Calais, France. E-mail: elias.ould-said@univ-littoral.fr 
Many types of dependency are considered in the literature. In this article, we consider the $\alpha$-mixing condition, which is defined hereafter:

Definition 1. Let $\left(U_{i}\right)_{i \geq 1}$ denote a sequence of stationary random variables ( $r v$ )'s and consider the family of $\sigma$-fields $\mathcal{F}_{j}^{k}=\left\{U_{i}, j \leq i \leq k\right\}$, Given a positive integer $n$, set

$$
\alpha(n)=\sup \left\{|\mathbb{P}(A \cap B)-\mathbb{P}(A) \mathbb{P}(B)|: A \in \mathcal{F}_{1}^{k} \text { and } B \in \mathcal{F}_{k+n}^{\infty}, k \in \mathbb{N}^{\star}\right\} .
$$

The sequence $\left(U_{i}\right)_{i \geq 1}$ is said to be $\alpha$-mixing (strong mixing) if the mixing coefficient $\alpha(n) \rightarrow 0$ as $n \rightarrow \infty$.

In what follows, we suppose that $\left(T_{i}\right)_{i \geq 1}$ and $\left(C_{i}\right)_{i \geq 1}$ are two independent sequences of stationary strongmixing rv's with mixing coefficients $\alpha_{1}(n)$ and $\alpha_{2}(n)$ respectively. [5] showed that $\left(Y_{i}\right)_{i \geq 1}$ is strongly mixing with coefficient $\alpha(n)=4 \max \left(\alpha_{1}(n), \alpha_{2}(n)\right)$. Consequently, we suppose that $\left(Y_{i}, \delta_{i}, X_{i}\right)_{i \geq 1}$ is strong mixing with a mixing coefficient equal to $\alpha$.

In the context of dependent observations, there is a large literature about the nonparametric estimation, $[9,10]$ considered the estimation of the mean regression and the quantile regression functions and $[14,15]$ showed respectively the uniform strong consistency and the asymptotic normality of the regression function. For more references concerning the nonparametric estimation with dependent data, see [12]. A censored real data example which satisfied the $\alpha$-mixing condition can be found in [27].

When a regression model is used for prediction the size of the error is of interest. What about when the data contains outliers, we know that the mean function is very sensible to outliers. This kind of data are usually encountered in medical and financial follow up studies. Hence, we consider the mean square relative error as loss function defined for all $T>0$ and $x \in \mathbb{R}^{d}$ by

$$
\mathbb{E}\left[T^{-2}(T-m(x))^{2} \mid X=x\right] .
$$

[23] studied (1) and derive an expression for the predictor $m(x)$ in terms of conditional inverse moments

$$
m(x)=\frac{\int t^{-1} f_{T \mid X}(t \mid x) d t}{\int t^{-2} f_{T \mid X}(t \mid x) d t}=: \frac{m_{1}(x)}{m_{2}(x)}
$$

where $f_{T \mid X}(\cdot \cdot \cdot)$ is the conditional density function of the couple $(X, T)$. The advantage of using the mean squared relative error as loss function is that is robust to outliers (see : [16], [18] and [23]).

Having in mind such kind of data, [20] extend the work of [13] to the case of a relative error regression (RER) model. They established the uniform strong consistency and the asymptotic normality of the RER function estimator. However, no simulation study has been done to comfort the theoretical result. We follow their approach, except that we assume that the data are dependent which is a very common case in practice.

Our major objective in this paper is to study the asymptotic propriety of $m(\cdot)$ uniformly in $x$ under strong mixing condition. This study extends the result of [20] to dependent data and constitutes a continuation result of [3] dealing with the uniform almost sure consistency of the RER estimator studied here.

The purpose of this paper is twofold. Section 2 is devoted to introduce the RER kernel estimate under right censoring and strong mixing condition. Section 3 contains the assumptions and main results. The first contribution of this paper is a consistency in probability result. Then, we establish an asymptotic normality result. Section 4 is dedicated to a numerical study on generated and real data sets. The proofs and auxiliary results are given in Section 5.

\section{Presentation of the model}

Consider $\left\{T_{i}, 1 \leq i \leq n\right\}$ be a sequence of true survival times for $n$ individuals. Let the survival time $T_{i}$ be censored on the right by the censoring time $C_{i}$ with unknown $\mathrm{df} G(c)=\mathbb{P}\left(C_{i} \leq c\right)$. So, we observe only 
$Y_{i}=\min \left(T_{i}, C_{i}\right)$ and $\delta_{i}=\mathbb{1}_{\left\{T_{i} \leq C_{i}\right\}}$ where $\delta_{i}$ the censoring index which takes a value of 0 if $T_{i}$ is censored and a value of 1 if we observe a variable of interest. Now let $X=\left(X_{1}, \cdots, X_{d}\right)$ be a vector of $d$-dimensional covariates with the marginal density function $f(x):=f_{X}(x)$ where $x=\left(x_{1}, \ldots, x_{d}\right)$. The data consist of $n$ replications $\left(Y_{i}, \delta_{i}, X_{i}\right)$ of the triplet $(Y, \delta, X)$. Throughout this article, we assume that $\left(C_{i}\right)_{i}$ and $\left(X_{i}, T_{i}\right)_{i}$ are independent. Now, to consider the censoring effect, we define, for $\gamma=1$, 2, the so-called synthetic data given by

$$
\widetilde{T}_{i}^{-\gamma}=\frac{\delta_{i} Y_{i}^{-\gamma}}{\bar{G}\left(Y_{i}\right)}, \quad 1 \leq i \leq n .
$$

Using the conditional expectation property and the independence assumption, for $\gamma=1,2$ and for all $x \in \mathbb{R}^{d}$, we get

$$
\begin{aligned}
\mathbb{E}\left[\widetilde{T}_{1}^{-\gamma} \mid X_{1}=x\right] & =\mathbb{E}\left[\frac{\delta_{1} Y_{1}^{-\gamma}}{\bar{G}\left(Y_{1}\right)} \mid X_{1}=x\right] \\
& =\mathbb{E}\left[\frac{T_{1}^{-\gamma}}{\bar{G}\left(T_{1}\right)} \mathbb{E}\left[\mathbb{1}_{\left\{T_{1} \leq C_{1}\right\}} \mid T_{1}\right] \mid X_{1}=x\right] \\
& =m_{\gamma}(x) .
\end{aligned}
$$

Consider now the "pseudo"-estimator of $m(\cdot)$ given by

$$
\widetilde{m}(x)=: \frac{\widetilde{m}_{1}(x)}{\widetilde{m}_{2}(x)}
$$

with

$$
\widetilde{m}_{\gamma}(x):=\frac{\frac{1}{n h_{n}^{d}} \sum_{i=1}^{n} \widetilde{T}_{i}^{-\gamma} K_{d}\left(\frac{x-X_{i}}{h_{n}}\right)}{\frac{1}{n h_{n}^{d}} \sum_{i=1}^{n} K_{d}\left(\frac{x-X_{i}}{h_{n}}\right)}=: \frac{\widetilde{\mu}_{\gamma}(x)}{\widehat{f}(x)},
$$

for $\gamma=1$, 2, where the kernel $K_{d}(\cdot)$ is a d-variate density function and $h_{n}>0$ is a smoothing parameter converging to zero as the sample size rises. Furthermore, $\widehat{f}(\cdot)$ is the kernel density function estimator defined by [25].

In practice $\bar{G}(\cdot)$ is unknown. Therefore, in order to define a genuine estimator of $m(\cdot)$, we replace $\bar{G}(\cdot)$ by its [19] estimate $\bar{G}_{n}(\cdot)$ given by

$$
\bar{G}_{n}(t)=\left\{\begin{array}{cc}
\prod_{i=1}^{n}\left(1-\frac{1-\delta_{(i)}}{n-i+1}\right)^{\left.\mathbb{1}\left\{Y_{(i)}\right)^{t}\right\}} & \text { if } t<Y_{(n)} \\
0 & \text { otherwise, }
\end{array}\right.
$$

where $Y_{(1)}<Y_{(2)}<\cdots<Y_{(n)}$ are the order statistics of the $Y_{i}$ 's and $\delta_{(i)}$ are the concomitant indicator of non-censoring. Then, (3) becomes for $\gamma=1,2$

$$
\widehat{T}_{i}^{-\gamma}=\frac{\delta_{i} Y_{i}^{-\gamma}}{\bar{G}_{n}\left(Y_{i}\right)}, \quad 1 \leq i \leq n .
$$

Therefore, replacing (6) in (4) we get the following estimator of (2):

$$
\widehat{m}(x):=\frac{\widehat{m}_{1}(x)}{\widehat{m}_{2}(x)}
$$

with

$$
\widehat{m}_{\gamma}(x):=\frac{\frac{1}{n h_{n}^{d}} \sum_{i=1}^{n} \widehat{T}_{i}^{-\gamma} K_{d}\left(\frac{x-X_{i}}{h_{n}}\right)}{\frac{1}{n h_{n}^{d}} \sum_{i=1}^{n} K_{d}\left(\frac{x-X_{i}}{h_{n}}\right)}=: \frac{\widehat{\mu}_{\gamma}(x)}{\widehat{f}(x)}
$$


for $\gamma=1$, 2. This is a proper representation of $m(x)$ in terms of estimable quantities. Note that this estimator is in the same spirit as the one proposed by [20], who worked under the independence and identically distributed data assumption. Therefore, if we take $\gamma=-1$ in formula (8), the estimator $\widehat{m}_{\gamma}(x)$ reduce to the ones proposed in $[14,15]$. Furthermore, when there is no censoring (all $\delta$ 's are equal to 1 ) the RER estimator in (7) reduces to [18] in the uni-dimensional case.

Remark 1. Among various mixing conditions used in the literature, $\alpha$-mixing is reasonably weak and has many practical applications see [7] and [5, 6]. In particular, the stationary autoregressive-moving average (ARMA) processes, which are widely applied in time series analysis, are $\alpha$-mixing. with an exponential decreasing rate. When the innovations satisfying some moment conditions, the sequence is geometrically strongly-mixing (see : [17]).

\section{Assumptions and main results}

To formulate our assumptions, some additional notations are required. Let $\mathcal{S}$ be a compact set of $\mathcal{S}_{0}:=\{x \in$ $\left.\mathbb{R}^{d} \mid f(x)>0\right\}$. For any df $F$ Let $\tau_{F}=\sup \{x, \bar{F}(x)>0\}$ be the upper bound of the support of $F$ and we assume that $\tau<\tau_{F}<\infty$ and then $0<\bar{G}\left(\tau_{F}\right)<\bar{G}(\tau)$.

Throughout the paper, $C, C_{1}, C_{2}, C_{3}, \cdots$, denote some positive constants not depending on $n$, which may be different in various places. Let us denote by

$$
\mu_{\gamma}(x)=\int t^{-\gamma} f_{X, T}(x, t) d t, \quad \gamma=\{1,2\}
$$

and

$$
r_{\ell}(x)=\int \frac{t^{-\ell}}{\bar{G}(t)} f_{X, T}(x, t) d t, \quad \ell=\{2,3,4\}
$$

where $f_{X, T}(\cdot, \cdot)=: f(\cdot, \cdot)$ is the joint density function of the couple $(X, T)$.

\subsection{Assumptions}

The assumptions used in this article are listed below. At this point, it is mentioned that all limits are taken as $n \rightarrow \infty$, unless otherwise specified.

(K) The kernel $K_{d}(\cdot)$ is a continuously differentiable compactly supported density function.

(i) $\int u_{j} K_{d}(u) d u_{j}=0$, for $1 \leq j \leq d$ and $u=\left(u_{1}, \cdots, u_{d}\right)^{t}$.

(ii) $\int\|u\| K_{d}^{2}(u) d u<\infty$ with $\|u\|=\sum_{i=1}^{n}\left|u_{i}\right|$.

(iii) $\int\left|u_{1} u_{2} \cdots u_{d}\right| K_{d}(u) d u<\infty$.

(D) The functions

(i) $\mu_{\gamma}(\cdot)$, for $\gamma=1,2$, are twice continuously differentiable.

(ii) $\mu_{\gamma}(\cdot, \cdot)$, for $\gamma=1,2$ exist and satisfy for all $(u, v) \in \mathbb{R}^{2 d}$

$$
\sup _{i, j} \sup _{u, v}\left|\mu_{i, j, \gamma}(u, v)-\mu_{i, \gamma}(u) \mu_{j, \gamma}(v)\right| \leq C<\infty .
$$

(iii) $r_{\ell}(x)$, for $\ell=2,3,4$, are continuously differentiable.

(iv) $f(\cdot)$ is continuously differentiable.

(M) $\left(T_{n}, X_{n}\right)_{n \geq 1}$ is a stationary $\alpha$-mixing sequence of rv's, with $\alpha(n)$. Furthermore, the mixing coefficient satisfies: 
(i) $\alpha(n)=\mathrm{O}\left(n^{-v}\right)$ for some $v>3$.

(ii) $h^{-\frac{d}{a_{1}}} \sum_{s>v_{n}} \alpha^{\frac{1}{a_{1}}}(s) \leq \infty$ for $0<a_{1}<1$ and $d>1$.

where $v_{n}$ is a sequence of positive integers going to infinity which will be defined later.

(H) The sequence $h_{n}$ satisfies:

(i) $\lim n h_{n}^{d+2}=0$,

(ii) $\lim h_{n}^{d} \log \log n=0$.

(B) There exist integer sequences $p_{n}$ and $q_{n}$ going to $\infty$ along with $n$ such that :

$$
\frac{p_{n}}{q_{n}} \rightarrow \infty, \quad \frac{k\left(p_{n}+q_{n}\right)}{n} \rightarrow 1, \quad \frac{p_{n}}{\sqrt{n h_{n}^{d}}} \rightarrow 0 \quad \text { and } \quad k \alpha\left(q_{n}\right) \rightarrow 0 .
$$

Moreover, for $k:=k_{n}=\left[\frac{n}{p_{n}+q_{n}}\right]$ (where [·] stands for the integer part function), we have

$$
k q_{n}^{-v} \longrightarrow 0
$$

\subsection{Discussion on the assumptions:}

First, the assumptions on the kernel (K) are standard in nonparametric estimation and assumptions (D) are regularity conditions which permit to get our result. The assumption $(\mathrm{H})$ about the bandwidth are reduced to the strict minimum to make some terms negligible (see Lemma 1). These three assumptions intervene in Lemmas 1-3 and corollaries 2 and 3.. The assumption (B) intervenes in Lemma 4, for using Doob's technique and established the asymptotic normality of the main part and makes the other terms negligible. The assumptions (M) are technical and concern the mixing coefficient $\alpha$.

The following result states the convergence in probability of the RER estimator.

Theorem 1. Suppose that the assumptions (K), (D)(ii, iii) and (M)(i) holds. We have

$$
\forall \varepsilon>0, \quad \lim _{n \rightarrow \infty} \mathbb{P}(|\widehat{m}(x)-m(x)|>\epsilon)=0 .
$$

The following result gives the asymptotic normality of the RER function estimator. Let $\mathcal{S}^{\star}=\{\forall x \in$ $\left.\mathcal{S}, r_{2}(x) \mu_{2}^{2}(x)-2 \mu_{1}(x) \mu_{2}(x) r_{3}(x)+\mu_{1}^{2}(x) r_{4}(x) \neq 0\right\}$.

Theorem 2. Under assumptions (B), (H), (M), (D) and (K), we have

$$
\left(\frac{n h_{n}^{d}}{\sigma^{2}(x)}\right)^{1 / 2}(\widehat{m}(x)-m(x)) \stackrel{\mathcal{D}}{\longrightarrow} \mathcal{N}(0,1), \quad \text { for all } x \in \mathcal{S}^{*}
$$

where

$$
\sigma^{2}(x)=\frac{r_{2}(x) \mu_{2}^{2}(x)-2 \mu_{1}(x) \mu_{2}(x) r_{3}(x)+\mu_{1}^{2}(x) r_{4}(x)}{\mu_{2}^{4}(x)} \int_{\mathbb{R}^{d}} K_{d}^{2}(s) d s
$$

with $\stackrel{\mathcal{D}}{\longrightarrow}$ denotes the convergence in distribution.

\subsection{Pointwise confidence intervals}

In nonparametric estimation, the asymptotic variance depends on certain unknown functions. In our case, to determine the pointwise confidences intervals, we have to estimate the unknown quantity (10), that appears 
in the asymptotic variance expression. Let us define a consistent estimator of $r_{\ell}(\cdot)$ for $\ell=2,3,4$ and for all $x \in \mathbb{R}^{d}$ by

$$
\widehat{r}_{\ell}(x)=\frac{1}{n h_{n}^{d}} \sum_{i=1}^{n} \frac{\delta_{i} Y_{i}^{-\ell}}{\bar{G}_{n}^{2}\left(Y_{i}\right)} K_{d}\left(\frac{x-X_{i}}{h_{n}}\right) .
$$

Then, we replace (12) in (11) we get a calculable estimator :

$$
\widehat{\sigma}^{2}(x)=\frac{\widehat{r}_{2}(x) \widehat{\mu}_{2}^{2}(x)-2 \widehat{\mu}_{1}(x) \widehat{\mu}_{2}(x) \widehat{r}_{3}(x)+\widehat{\mu}_{1}^{2}(x) \widehat{r}_{4}(x)}{\widehat{\mu}_{2}^{4}(x)} \int_{\mathbb{R}^{d}} K_{d}^{2}(s) d s .
$$

Finally, Theorem 2 becomes

Corollary 1. Under the assumptions of Theorem 2, we have

$$
\left(\frac{n h_{n}^{d}}{\widehat{\sigma}^{2}(x)}\right)^{1 / 2}(\widehat{m}(x)-m(x)) \stackrel{\mathcal{D}}{\longrightarrow} \mathcal{N}(0,1) .
$$

The confidence intervals of level $0<\beta<1$ are giving by

$$
] \widehat{m}(x)-z_{1-\frac{\beta}{2}}\left(\frac{\widehat{\sigma}^{2}(x)}{n h_{n}^{d}}\right)^{1 / 2} ; \widehat{m}(x)+z_{1-\frac{\beta}{2}}\left(\frac{\widehat{\sigma}^{2}(x)}{n h_{n}^{d}}\right)^{1 / 2}[
$$

where $z_{1-\frac{\beta}{2}}$ stands for the quantile of the standard normal distribution.

\section{Simulations}

The goal of this section is to examine the performance of $\widehat{m}(\cdot)$. A generated data illustration of our RER estimator's asymptotic normality is done. We then compare the shape of their estimated density with normalized deviation with respect to the standard normal density. To this aim, two stationary strong mixing processes were considered in this study :

$$
\text { Model 1: }\left\{\begin{array}{rl}
X_{i} & =\rho_{1} X_{i-1}+\sqrt{1-\rho_{1}^{2}} \epsilon_{i} \\
T_{i} & =\frac{1}{2} X_{i+1}+2
\end{array} \quad i=1, \cdots, n .\right.
$$

and

$$
\text { Model 2: }\left\{\begin{array}{rl}
X_{i} & =\rho_{1} X_{i-1}+\rho_{2} X_{i-2}+\epsilon_{i} \\
T_{i} & =X_{i+1}+4
\end{array} \quad i=1, \cdots, n .\right.
$$

where $0 \leq \rho_{1}, \rho_{2} \leq 1$ and $\epsilon_{i}$ is a white noise sequence and $X_{0}, X_{1} \rightsquigarrow \mathcal{N}(0,1)$. On the one hand, the model 1 is an (auto-regressive) AR(1) of theoretical function given by

$$
m(x)=\frac{\rho_{1}}{2} x+2 .
$$

On the other hand, the model 2 is an $\mathrm{AR}(2)$ process of theoretical function given by

$$
m(x)=\left(\rho_{1}+\rho_{2}\right) x+4,
$$

for all $x \in[1,4]$. We simulate $\left(C_{i}\right)_{i}$ as an exponential law (i.e. with density $\left.\lambda \exp (-\lambda x) \mathbb{1}_{\{x \geq 0\}}\right)$ where the parameter $\lambda$ is a varying constant which allows us to adapt the censoring rate (CR). The simulations are carried out for samples size $n=100, n=200$ and $n=400$ and the results are obtained by using $L=200$ replications. We calculate the following quantities:

$$
\begin{aligned}
& \text { Model 1: } \chi_{j}:=\sqrt{\frac{n h_{n}}{\widehat{\sigma}^{2}(0)}}\left(\widehat{m}_{j}(0)-2\right) \\
& \text { Model 2: } \omega_{j}:=\sqrt{\frac{n h_{n}}{\widehat{\sigma}^{2}(0)}}\left(\widehat{m}_{j}(0)-4\right), \quad \text { for } 1 \leq j \leq L .
\end{aligned}
$$


where $\widehat{\sigma}^{2}(0)$ is computed from the formula (13) at $x=0$. For the weights that appear in our estimator $\widehat{m}(x)$, we use the standard Gaussian kernel function $\left(K(u)=\frac{1}{\sqrt{2 \pi}} \exp \left(-\frac{u^{2}}{2}\right)\right)$. In this study, three values of the parameters $\rho_{1}$ and $\rho_{2}$ are investigated, $\rho_{i}=0.3,0.6$ and 0.9 for $i=1,2$ (where 0.9 is a strong dependency). For each figure, we use a different bandwidth $h_{n}$ that we select over a grid of 200 equidistant values of $h_{n}$ in the grid 0.01 to 2 and we take the value that minimizes the cross-validation criterion (see the remark below). We construct for $\chi_{j}$ and $\omega_{j}$ where $1 \leq j \leq L$ a kernel estimator of the normal standard density, see [25]. It is wellknown, that to estimate the density function, we choose $h^{\star}=C L^{-0.2}$ where the constant $C$ is appropriately chosen, see [26].

Remark 2. The practical performance of a nonparametric regression model depend strongly on the smoothing parameter. Choosing an optimal $h_{n}$ is often problematic. In order to obtain a satisfactory results, it is clear that an appropriate value of $h_{n}$ is very important as it influence the behavior of the predictor. We use the crossvalidation method to determine the optimal bandwidth. In our case of RER estimation, it remains to minimize the mean square of the relative prediction error. One may use the following criterion:

$$
C V_{h_{n}}(x)=\frac{1}{n-1} \sum_{i=1}^{n}\left(\frac{T_{i}-\widehat{m}_{h_{n}}^{-i}(x)}{T_{i}}\right)^{2}
$$

where $\widehat{m}_{h_{n}}^{-i}(\cdot)$ is the RER estimator defined in (7) suppressing the triplet observation $\left(X_{i}, Y_{i}, \delta_{i}\right), i=1, \ldots, n$.

\subsection{Model 1}

\subsubsection{Effect of sample size}

From Fig. 1, we can see that the quality of fit becomes better when $n$ increases. However, we have remarked that as the percentage of censoring increases, one needs a large sample to get better results.
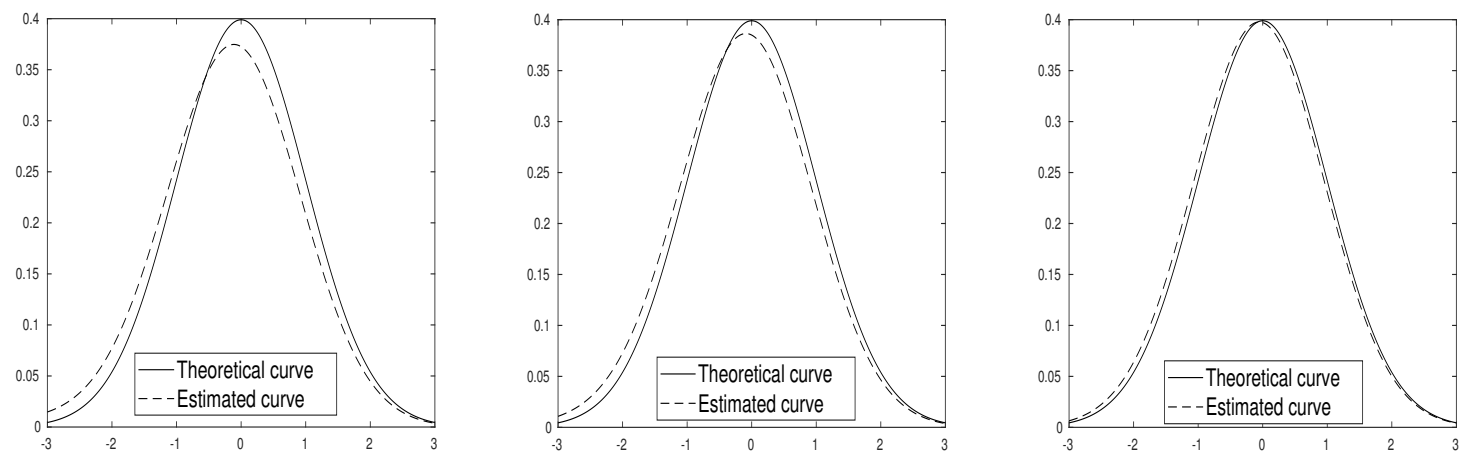

Figure 1: $C R \approx 55 \%$ and $\rho=0.3$ for $n=50,200$ and 400 respectively.

\subsubsection{Effect of dependency}

From Fig. 2, as predicted when $\rho_{1}$ increases the quality of fit deteriorates a little bit. However, we see clearly that it remains well. 

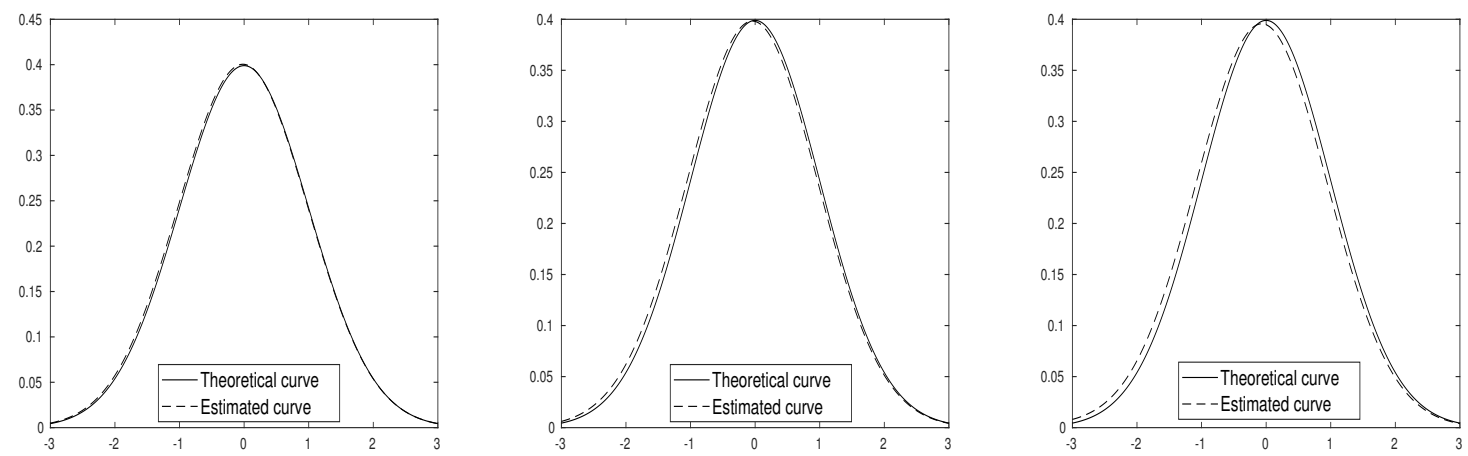

Figure 2: $n=200$ and $\mathrm{CR} \approx 55 \%$ for $\rho_{1}=0.3,0.6$ and 0.9 respectively.

\subsubsection{Effect of censoring}

As expected, we can see from Fig. 3 the quality of the estimator decreases with increasing censoring. This can be explained by the fact that we observe less true values.
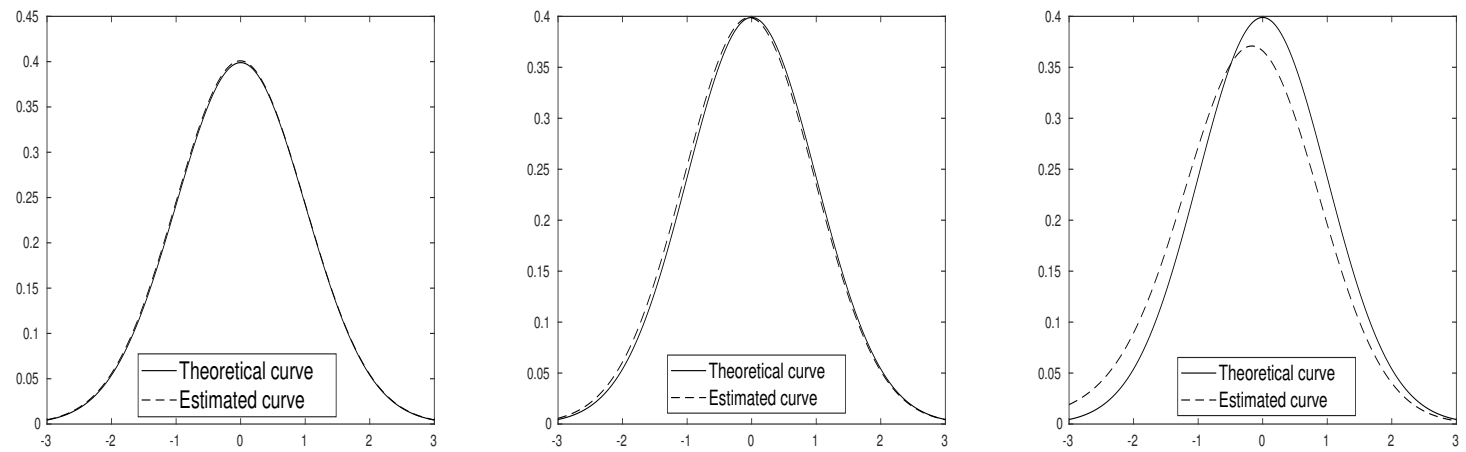

Figure 3: $n=200$ and $\rho=0.6$ for $C R \approx 18,54$ and $80 \%$ respectively.

\subsection{Model 2}

\subsubsection{Effect of sample size}

As claimed in Fig 1 , the quality of fit to $\mathcal{N}(0,1)$ curve becomes better when $n$ rises. We can also remark that there is no significant difference in the performance between model 1 and model 2 (see Fig. 4). 

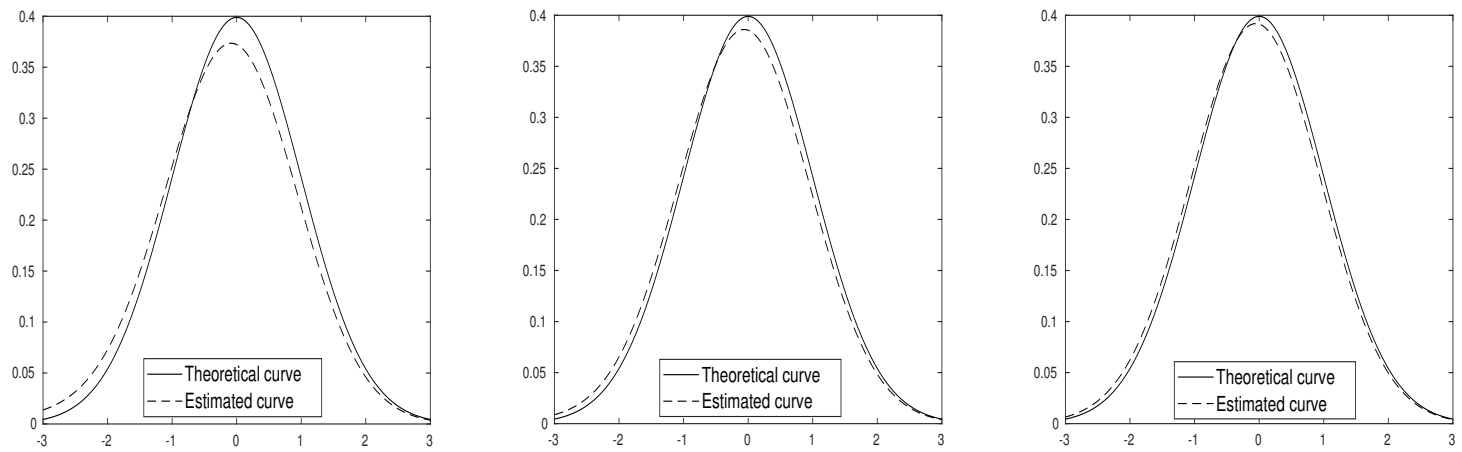

Figure 4: $\mathrm{CR} \approx 48 \%$ and $\left(\rho_{1}, \rho_{2}\right)=(0.3,0.3)$ for $n=50,200$ and 400 respectively.

\subsubsection{Effect of censoring}

From 5, we can observe that when the CR is small the theoretical and estimated curves are very close. However, when the CR is high (83\%), the estimated curve deviates from the theoretical one taking into account that $\rho_{2}=0.8$ which is predictable.
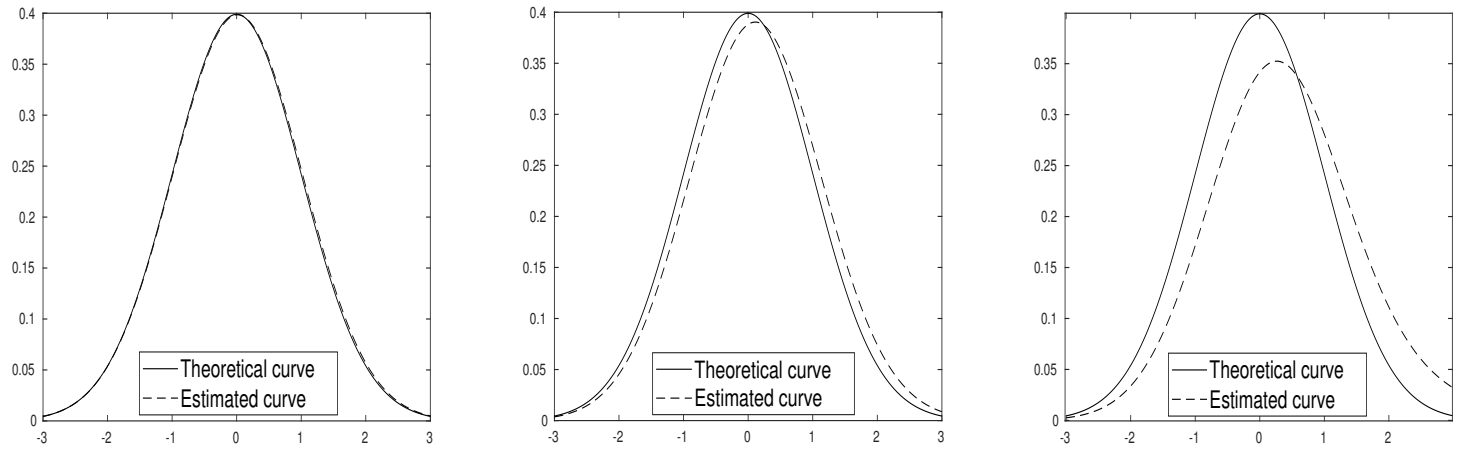

Figure 5: $n=200$ and $\left(\rho_{1}, \rho_{2}\right)=(0.1,0.8)$ for $C R \approx 12,42,83 \%$ respectively.

\subsubsection{Effect of dependency}

As we can see from Fig. 6, the Gaussian standard normality estimator is slightly affected by the degree of dependency in data even if we only observe almost half of the sample size. Generally, the instability increases as both $\rho_{1}, \rho_{2}$ tend 1 .

So, we can conclude that the main variation in the quality of the proposed estimator is connected with strong censoring and dependency levels. 

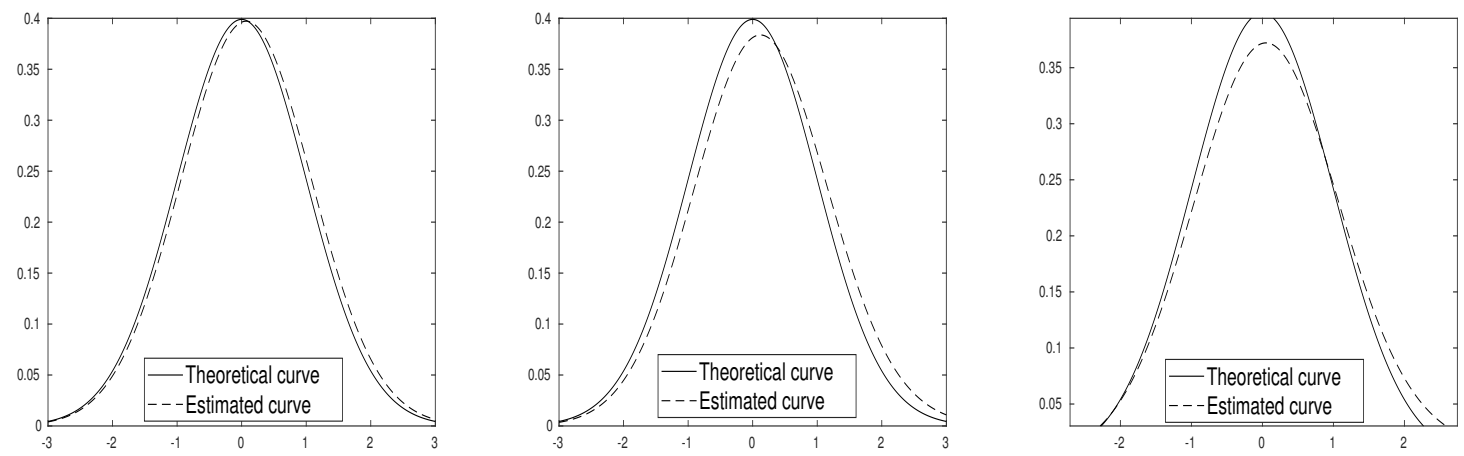

Figure 6: $n=200$ and $C R \approx 46 \%$ for $\left(\rho_{1}, \rho_{2}\right)=(0.1,0.1),(0.2,0.5)$ and $(0.3,0.8)$ respectively.

\subsection{Coverage probability}

In the following table, we have the coverage probabilities for confidence levels $1-\beta$ where $\beta=0.01$ and 0.05 . We performed 100 replications of sample size $n=50,200$ and 400 with different censoring rates CR $\approx 10,30$ and 50 and dependency levels $\rho_{1}=0.3$ and 0.9 . Moreover, as we stated that our estimator is robust to the presence of outliers in the sample. We generate outliers in the sample by multiplying a certain number of observed data $Y_{i}$ for $\left\{i=20 \times \iota\right.$ with $\left.1 \leq \iota \leq \frac{n}{20}\right\}$ by a multiplying coefficient (MC) that we vary. In order to calculate the coverage probabilities, we observe the proportion of times that the confidence intervals contains the true regression function. We would like to mention that the model used here is model 1 . After analyzing and comparing, we have the following remarks. Note that, for $n=50$ and $\rho_{1}=0.3$ the coverage probabilities are equal to 1 expected two cases $(\mathrm{CR} \approx 30 \%, \beta=0.05, \mathrm{MC}=80)$ and $(\mathrm{CR} \approx 50 \%, \beta=0.01, \mathrm{MC}=10)$. The justification for this comes from the fact that for the first one, the $\mathrm{MC}$ is high and for the second one the $\mathrm{CR}$ is high. Regarding the dependence in data, by comparing for $\rho_{1}=0.3$ and $\rho_{1}=0.9$ (which means comparing between weak and strong dependency), we can see that $\rho_{1}$ has relative effect on the results depending on the other parameters. Furthermore, we can see that the MC has no significant influence on the coverage probabilities. This may be explained by the robustness of the relative regression to outliers. The latter remains an important element of table 1. It is clear that as the sample size increases, the probability that the interval contains the true value becomes small due to the fact that the width of the intervals reduces. Another interesting remark related to dependency is the fact that with $\rho_{1}=0.9$, the proportion of observed true values decreases which is normal because that data are in a strong dependence case. Obviously, the RER estimator behaves better under weak dependency.

Remark 3. Regarding the number of replications, it is not very high, this is due only for the duration of the calculations and the fact that there are many loops in the program. Furthermore, we see clearly, that as the sample size increases and in the strong dependency the recovery decreases. We point out that coverage remains stable with respect to high outliers, which is one of the advantages of the relative error method.

\subsection{Pointwise confidence interval}

In this subsection, we implement our methodology and see how good is the asymptotic normality in the determination of confidences bands. To this purpose, we consider the model 1 and construct a 95\%-confidence curves given in corollary 1 . On the four figures that follow, we display the theoretical function 14 and the RER function estimator $\widehat{m}(x)$ for $x \in[1,4]$. The kernel and the bandwidth are chosen as above. 
Table 1: Coverage probabilities for 100 replications.

\begin{tabular}{|c|c|c|c|c|c|c|c|c|}
\hline \multirow[b]{2}{*}{$\mathrm{CR}(\approx \%)$} & \multirow[b]{2}{*}{$\beta$} & \multirow[b]{2}{*}{ MC } & \multicolumn{2}{|c|}{$n=50$} & \multicolumn{2}{|c|}{$n=200$} & \multicolumn{2}{|c|}{$n=400$} \\
\hline & & & $\rho_{1}=0.3$ & $\rho_{1}=0.9$ & $\rho_{1}=0.3$ & $\rho_{1}=0.9$ & $\rho_{1}=0.3$ & $\rho_{1}=0.9$ \\
\hline \multirow{6}{*}{10} & \multirow{3}{*}{0.05} & 10 & 1.0000 & 0.8321 & 0.9996 & 0.9148 & 0.8774 & 0.7749 \\
\hline & & 40 & 1.0000 & 0.8296 & 0.9975 & 0.9112 & 0.8742 & 0.7623 \\
\hline & & 80 & 1.0000 & 0.8141 & 0.9971 & 0.8939 & 0.8670 & 0.7485 \\
\hline & \multirow{3}{*}{0.01} & 10 & 1.0000 & 0.8540 & 0.9995 & 0.9148 & 0.9077 & 0.8022 \\
\hline & & 40 & 1.0000 & 0.8499 & 0.9994 & 0.8905 & 0.8973 & 0.8009 \\
\hline & & 80 & 1.0000 & 0.8354 & 0.9992 & 0.8760 & 0.8932 & 0.7991 \\
\hline \multirow{6}{*}{30} & \multirow{3}{*}{0.05} & 10 & 1.0000 & 0.8224 & 0.9989 & 0.9047 & 0.8915 & $\overline{0.7926}$ \\
\hline & & 40 & 1.0000 & 0.8013 & 0.9974 & 0.9025 & 0.9807 & 0.7813 \\
\hline & & 80 & 0.9995 & 0.7448 & 0.9961 & 0.8402 & 0.8779 & 0.7439 \\
\hline & \multirow{3}{*}{0.01} & 10 & 1.0000 & 0.8327 & 0.9990 & 0.9355 & 0.9216 & 0.8130 \\
\hline & & 40 & 1.0000 & 0.8187 & 0.9993 & 0.9204 & 0.9155 & 0.8048 \\
\hline & & 80 & 1.0000 & 0.8172 & 0.9993 & 0.8875 & 0.8979 & 0.7757 \\
\hline \multirow{6}{*}{50} & \multirow{3}{*}{0.05} & 10 & 1.0000 & 0.7770 & 0.9996 & 0.8620 & 0.9126 & 0.7850 \\
\hline & & 40 & 1.0000 & 0.7318 & 0.9994 & 0.8435 & 0.9090 & 0.6748 \\
\hline & & 80 & 1.0000 & 0.7251 & 0.9993 & 0.8327 & 0.9032 & 0.6665 \\
\hline & \multirow{3}{*}{0.01} & 10 & 0.9970 & 0.7987 & 0.9998 & 0.9025 & 0.9497 & 0.7961 \\
\hline & & 40 & 1.0000 & 0.7687 & 0.9994 & 0.9019 & 0.9381 & 0.7566 \\
\hline & & 80 & 1.0000 & 0.6823 & 0.9993 & 0.8476 & 0.9349 & 0.7309 \\
\hline
\end{tabular}

\subsubsection{Weak dependency and censoring}

Fig. 7 represents the "ideal" case where the data are weakly dependent and the censoring percentage is approximately $1 / 4$ of the sample size. Obviously, the confidence bands width decreases when $n$ increases which is predictable.
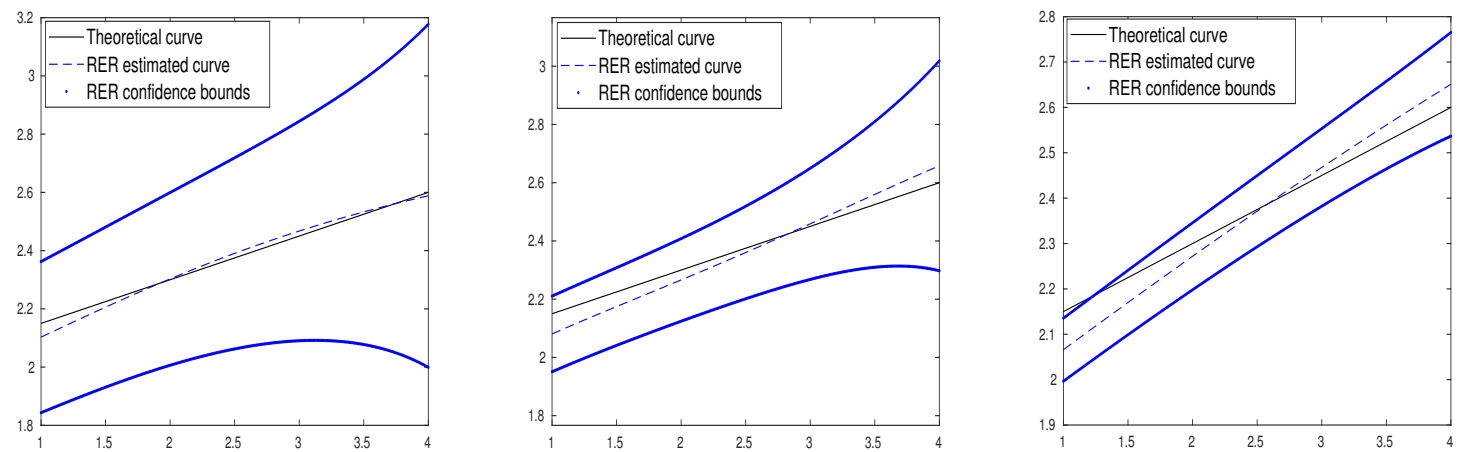

Figure 7: $\rho_{1}=0.3$ and $C R \approx 25 \%$ for $n=50,200$ and 400 respectively. 


\subsubsection{Weak dependency and strong censoring}

From Fig 8, we can see that for moderate dependency level $\left(\rho_{1}=0.3\right)$ and a strong percentage of censoring (85\%), the RER estimator behaves well. An interesting remark related to censoring is the fact that even with a high level of censoring the RER function estimator continues to track the theoretical curve.
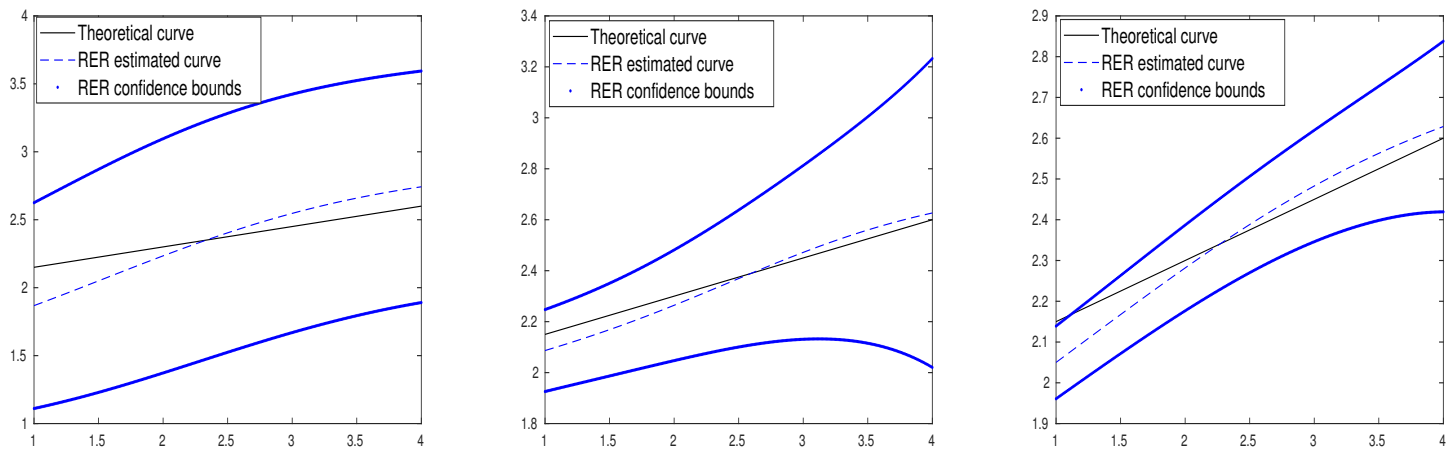

Figure 8: $\rho_{1}=0.3$ with $C R \approx 85 \%$ and $n=50,200$ and 400 respectively.

\subsubsection{Strong dependency and weak censoring}

Fig. 9 is the opposite case of Fig. 8. We can observe that the quality of adjustment becomes better when $n$ rises as well as the intervals decreases. It can be seen that the dependency in the data influences mostly the right edge. We can say the fact that the RER estimator remains resistant to a strong dependence.
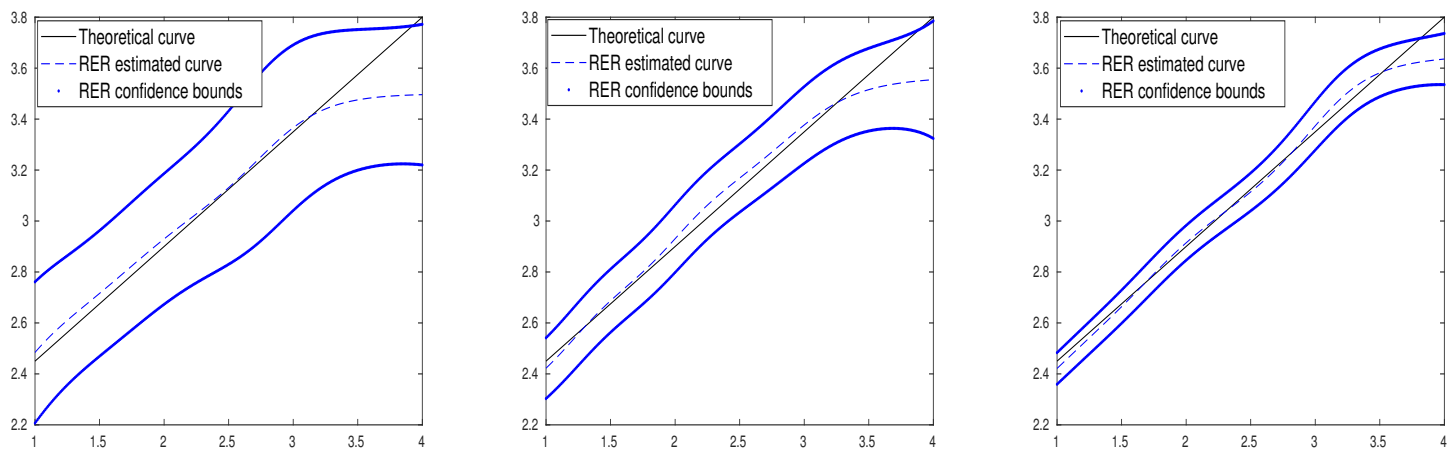

Figure 9: $\rho_{1}=0.9$ with $\mathrm{CR} \approx 25 \%$ and $n=50,200$ and 400 respectively.

\subsubsection{Strong dependency and censoring}

Fig. 10 displays the "worst" case which is a high levels of censoring $(85 \%)$ and dependency $\left(\rho_{1}=0.9\right)$. As we can see, the estimator deviates completely from the theoretical curve at the right edge. This is due to the high rate of censoring and dependence. This becomes more apparent as the intervals become smaller (when 
$n=400$ ). This can eventually be corrected by choosing the value of the risk $\beta$ at $1 \%$ which allows us to increase the interval. However we preferred to keep the same value of beta risk to be able comparing the results.
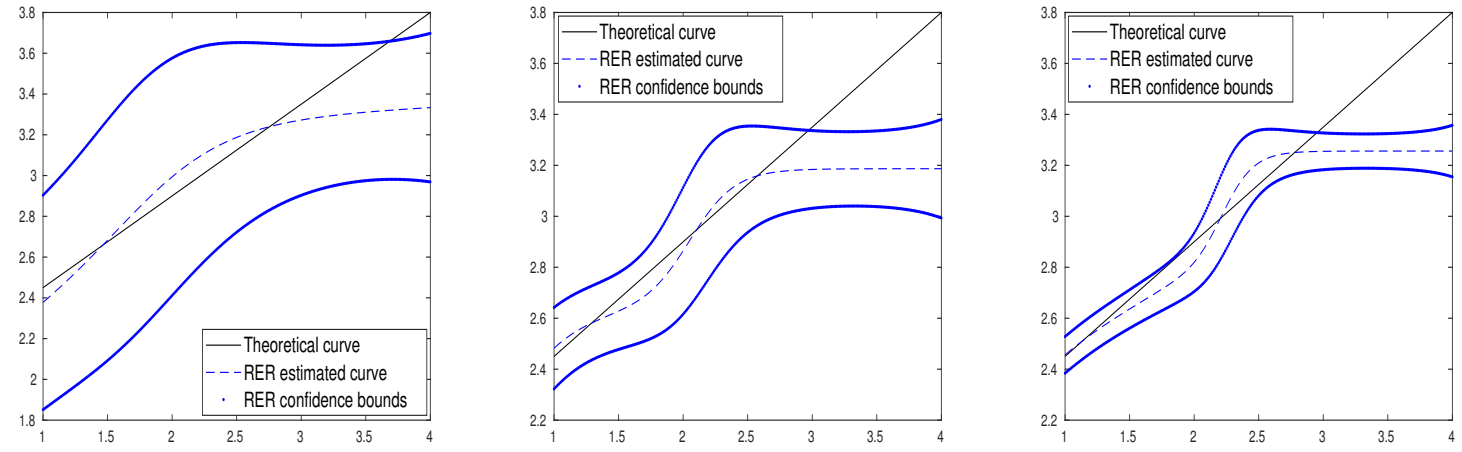

Figure 10: $\rho_{1}=0.9$ with $\mathrm{CR} \approx 85 \%$ and $n=50,200$ and 400 respectively.

\subsection{A real data study}

In this subsection, we analyze a real data set to illustrate the efficiency of the RER predictor in the presence of censored dependent data. The data consists on patients diagnosed with acquired immunodeficiency syndrome (AIDS) in Australia before $1^{\text {st }}$ July 1991. The source of data is Dr P.J. Solomon and the Australian National Center in HIV Epidemiology and Clinical Research. For more information about the data, see : [28]. A total of $N=2843$ patients have been taken into account where about 1770 had died by the end of the study. The file contains the dates of first diagnosis; the date of death or end of observation; the sex of patients; age (in year) at diagnosis; the status ("A" for alive or "D" for dead) at the end of observation; as well as the state and the coded transmission category. The factors that are expected to be important is the times to death and diagnosis. After calculating the covariance matrix, we found a strong correlation between time to diagnosis and time to death, (the correlation coefficient is equal to 0.8312 ) which permit to claim that the data dependent. So, by using the partial correlation plot, it appears that our data are an auto-regressive process of order greater than 1 . In the aim of prediction and pointwise $95 \%$-confidence bounds, we split the $N$-sample into two subsets. The learning sample of size $n=2400$ (i.e. $\left\{\left(Y_{i}, \delta_{i}, X_{i}\right), 1 \leq i \leq n\right\}$ ) will be our statistical sample for which our estimator is calculated. The test sample of size $n^{*}=443$ allows us to evaluate our predictions. In addition, the kernel function and the optimal bandwidth are selected as for generated data. The censoring rate is approximately equal to $62 \%$ in $N$-sample. Note that, we eliminate the censoring data from the predicted values (i.e. if for any $n+1 \leq i \leq N$ the observed variable $Y_{i}=C_{i}$, we remove the observation $Y_{i}$ from the predicted values. Obviously, censored data cannot be predicted. We forecast only the interest variables). We point out, the CR in the $N$-sample is different of the CR of $n^{\star}$-sample due that the selection is random. Then, only 13 values are predicted from the $n^{\star}$-sample. For the left hand of Fig. 11 we show a scatter plot of the data where we distinguish between the censored and uncensored data. For the right hand, we plot true, predicted and confidence bands. It can be seen that all true value are very near to predicted value and between the upper and lower bands. This shows that our method is robust. 

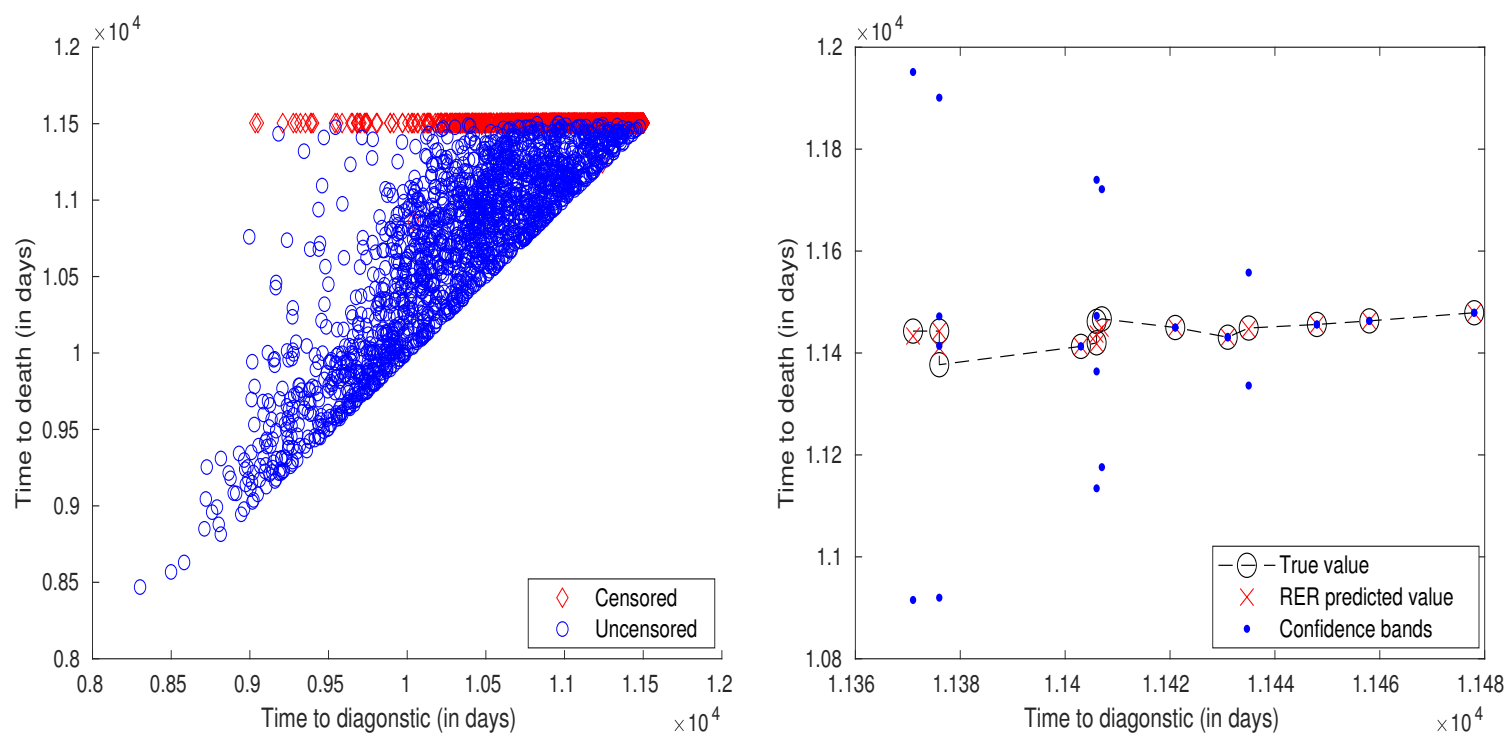

Figure 11: Australian AIDS survival scatter plot of censored / uncensored data. (left) RER prediction of survival time versus time to diagnosis for AIDS patients with pointwise interval confidence. (right)

\section{Appendix : Proofs}

For $x \in \mathbb{R}^{d}$, we consider the following decomposition :

$$
\widehat{m}(x)-m(x)=\frac{1}{\widehat{\mu}_{2}(x)}\left\{\sum_{1 \leq k \leq 3} \mathcal{B}_{k, 1}(x)-m(x) \sum_{1 \leq k \leq 3} \mathcal{B}_{k, 2}(x)\right\}
$$

where

$$
\begin{array}{lll}
\mathcal{B}_{1, \gamma}(x) & :=\widehat{\mu}_{\gamma}(x)-\widetilde{\mu}_{\gamma}(x), & \\
\mathcal{B}_{2, \gamma}(x):=\widetilde{\mu}_{\gamma}(x)-\mathbb{E}\left[\widetilde{\mu}_{\gamma}(x)\right], & \\
\mathcal{B}_{3, \gamma}(x):=\mathbb{E}\left[\widetilde{\mu}_{\gamma}(x)\right]-\mu_{\gamma}(x), & \text { for } \gamma=1,2 .
\end{array}
$$

Proof of Theorem 1. To establish the result, it suffices to show that each term in (16) tends to 0 in probability. For $\mathcal{B}_{1, \gamma}(x)$ and $\mathcal{B}_{3, \gamma}(x)$, we have from Lemma 1 and Lemma 2 in [3] that

$$
\mathcal{B}_{1, \gamma}(x)=\mathrm{O}_{a . s}\left(\sqrt{\frac{\log \log n}{n}}\right)
$$

and

$$
\mathcal{B}_{3, \gamma}(x)=\mathrm{O}\left(h_{n}\right) .
$$

We consider now the dominant term $\left\{\mathcal{B}_{2, \gamma}(x)\right.$, for $\left.\gamma=1,2\right\}$ and prove that

$$
\nu:=\operatorname{Var}\left(\tilde{\mu}_{\gamma}(x)\right) \longrightarrow 0 \text {. }
$$

We have

$$
\begin{aligned}
\mathcal{V} & =\frac{1}{\left(n h_{n}^{d}\right)^{2}}\left\{\sum_{i=1}^{n} \operatorname{Var}\left(\widetilde{T}_{i}^{-\gamma} K_{d}\left(\frac{x-X_{i}}{h_{n}}\right)\right)+\sum_{\substack{i, j=1 \\
i \neq j}}^{n} \operatorname{Cov}\left(\widetilde{T}_{i}^{-\gamma} K_{d}\left(\frac{x-X_{i}}{h_{n}}\right), \widetilde{T}_{j}^{-\gamma} K_{d}\left(\frac{x-X_{j}}{h_{n}}\right)\right)\right\} \\
& =\frac{1}{\left(n h_{n}^{d}\right)^{2}}\left\{\sum_{i=1}^{n} \mathcal{V}_{1}+\sum_{\substack{i, j=1 \\
i \neq j}}^{n} V_{2}\right\} .
\end{aligned}
$$


We have now to deal with $\mathcal{V}_{1}$; for this compute

$$
\begin{aligned}
\nu_{1} & =\mathbb{E}\left[\frac{\delta_{1} Y_{1}^{-2 \gamma}}{\bar{G}^{2}\left(Y_{1}\right)} K_{d}^{2}\left(\frac{x-X_{1}}{h_{n}}\right)\right]-\mathbb{E}^{2}\left[\widetilde{T}_{1}^{-\gamma} K_{d}\left(\frac{x-X_{1}}{h_{n}}\right)\right] \\
& =: V_{1,1}-V_{1,2} .
\end{aligned}
$$

For $\mathcal{V}_{1,1}$, let denote by $\{\ell=2 \gamma$ where $\gamma=1,2\}$. Then, using the conditional expectation property and a change of variable, we get

$$
\begin{aligned}
\nu_{1,1} & =\int K_{d}^{2}\left(\frac{x-u}{h_{n}}\right) \int \frac{t^{-\ell}}{\bar{G}(t)} f(t \mid u) d t f(u) d u \\
& =\int K_{d}^{2}\left(\frac{x-u}{h_{n}}\right) \int \frac{t^{-\ell}}{\bar{G}(t)} f(u, t) d t d u \\
& =h_{n}^{d} \int K_{d}^{2}(t) r_{\ell}\left(x-h_{n} t\right) d t .
\end{aligned}
$$

Using first order Taylor expansion for $\xi \in] x-h_{n} t, x[$, we have

$$
r_{\ell}\left(x-h_{n} t\right)=r_{\ell}(x)-h_{n} \sum_{i=1}^{d} t_{i} \frac{\partial r_{\ell}(\xi)}{\partial x_{i}} .
$$

Hence, under (D)(iii) and (K)(ii) we get

$$
\begin{aligned}
\left|\mathcal{V}_{1,1}\right| & =h_{n}^{d}\left|\int K_{d}^{2}(t)\left(r_{\ell}(x)-h_{n} \sum_{i=1}^{d} t_{i} \frac{\partial r_{\ell}(\xi)}{\partial x_{i}}\right) d t\right| \\
& \leq h_{n}^{d} \int K_{d}^{2}(t)\left(\left|r_{\ell}(x)\right|+h_{n}\left|\sum_{i=1}^{d} t_{i} \frac{\partial r_{\ell}(\xi)}{\partial x_{i}}\right|\right) d t \\
& =C_{1} \mathrm{O}\left(h_{n}^{d}\right) .
\end{aligned}
$$

For $\mathcal{V}_{1,2}$, using the conditional expectation property, a change of variable and second order Taylor development of $\mu_{\gamma}(\cdot)$, for $\gamma=1$, 2. Under (K)(ii, iii) and (D)(i) we get

$$
\begin{aligned}
\left|\mathcal{V}_{1,2}\right| & =\left|\left(\int K_{d}\left(\frac{x-u}{h_{n}}\right) \mu_{\gamma}(u) d u\right)^{2}\right| \\
& =h_{n}^{2 d}\left|\left(\int K_{d}(t) \mu_{\gamma}\left(x-h_{n} t\right) d t\right)^{2}\right| \\
& =h_{n}^{2 d}\left|\left(\mu_{\gamma}(x)+\frac{h_{n}^{2}}{2} \int K_{d}(t)\left\{\sum_{i=1}^{d} t_{i}^{2} \frac{\partial^{2} \mu_{\gamma}(\xi)}{\partial x_{i}^{2}}+2 \sum_{\substack { i, j=1 \\
\begin{subarray}{c}{i \neq j \\
j{ i , j = 1 \\
\begin{subarray} { c } { i \neq j \\
j } }\end{subarray}}^{d} t_{i} t_{j} \frac{\partial^{2} \mu_{\gamma}(\xi)}{\partial x_{i} \partial x_{j}}\right\} d t\right)^{2}\right| \\
& \left.\leq h_{n}^{2 d}|| \mu_{\gamma}(x) \mid+\frac{h_{n}^{2}}{2}\left\{\int K_{d}(t) \sum_{i=1}^{d} t_{i}^{2}\left|\frac{\partial^{2} \mu_{\gamma}(\xi)}{\partial x_{i}^{2}}\right| d t+2 \int K_{d}(t) \sum_{\substack{i, j=1 \\
i \neq j}}^{d}\left|t_{i} t_{j} \frac{\partial^{2} \mu_{\gamma}(\xi)}{\partial x_{i} \partial x_{j}}\right| d t\right\}\right)^{2} \\
& =C_{2} \mathrm{O}\left(h_{n}^{2 d}\right) .
\end{aligned}
$$

Then, combining (18) and (19) give

$$
\begin{aligned}
\left|\mathcal{V}_{1}\right| & \leq\left|\mathcal{V}_{1,1}\right|+\left|\mathcal{V}_{1,2}\right| \\
& =C_{1} \mathrm{O}\left(h_{n}^{d}\right)+C_{2} \mathrm{O}\left(h_{n}^{2 d}\right) \\
& =C_{3} \mathrm{O}\left(h_{n}^{d}\right) .
\end{aligned}
$$


Next, we will calculate the second term $v_{2}$. Using the conditional expectation property, a change of variable and under (K)(i) and (D)(i, ii) we obtain

$$
\begin{aligned}
\left|\mathcal{V}_{2}\right| & =\mid \mathbb{E}\left[\widetilde{T}_{1}^{-\gamma} \widetilde{T}_{2}^{-\gamma} K_{d}\left(\frac{x-X_{1}}{h_{n}}\right) K_{d}\left(\frac{x-X_{2}}{h_{n}}\right)\right] \\
& -\mathbb{E}\left[\widetilde{T}_{1}^{-\gamma} K_{d}\left(\frac{x-X_{1}}{h_{n}}\right)\right] \mathbb{E}\left[\widetilde{T}_{2}^{-\gamma} K_{d}\left(\frac{x-X_{2}}{h_{n}}\right)\right] \mid \\
& =\mid \iint K_{d}\left(\frac{x-u}{h_{n}}\right) K_{d}\left(\frac{x-v}{h_{n}}\right) \mu_{\gamma}(u, v) d u d v \\
& -\int K_{d}\left(\frac{x-u}{h_{n}}\right) \mu_{\gamma}(u) d u \int K_{d}\left(\frac{x-v}{h_{n}}\right) \mu_{\gamma}(v) d v \mid \\
& \leq \iint K_{d}\left(\frac{x-u}{h_{n}}\right) K_{d}\left(\frac{x-v}{h_{n}}\right)\left|\mu_{\gamma}(u, v)-\mu_{\gamma}(u) \mu_{\gamma}(v)\right| d u d v \\
& \leq h_{n}^{2 d} \iint K_{d}(t) K_{d}(s)\left|\mu_{\gamma}\left(x-h_{n} t, x-h_{n} s\right)-\mu_{\gamma}\left(x-h_{n} t\right) \mu_{\gamma}\left(x-h_{n} s\right)\right| d t d s \\
& =C_{4} \mathrm{O}\left(h_{n}^{2 d}\right) .
\end{aligned}
$$

Let introduce a sequence of integers $v_{n} \longrightarrow \infty$, so that

$$
\left(n h_{n}^{d}\right)^{-2} \sum_{\substack{1 \leq i, j \leq n \\ i \neq j}}\left|\mathcal{V}_{2}\right| \leq\left(n h_{n}^{d}\right)^{-2} \sum_{1 \leq|i-j| \leq v_{n}}\left|\mathcal{V}_{2}\right|+\left(n h_{n}^{d}\right)^{-2} \sum_{v_{n}<|i-j| \leq n}\left|\mathcal{V}_{2}\right|
$$

On the one hand, let $[x]$ is the integer part of $x$, by choosing $v_{n}=\left[h_{n}^{-d}\right]$, we get from (21)

$$
\begin{aligned}
\left(n h_{n}^{d}\right)^{-2} \sum_{1 \leq|i-j| \leq v_{n}}\left|\mathcal{V}_{2}\right| & \leq C_{5}\left(n h_{n}^{d}\right)^{-2} h_{n}^{2 d} n v_{n} \\
& =C_{5} \mathrm{O}\left(\left(n h_{n}^{d}\right)^{-1}\right) .
\end{aligned}
$$

On the other hand, from [2], we have $\left|\mathcal{V}_{2}\right| \leq C \alpha(|i-j|)$, then

$$
\begin{aligned}
\left(n h_{n}^{d}\right)^{-2} \sum_{v_{n}<|i-j| \leq n}\left|\mathcal{V}_{2}\right| & \leq C_{5}\left(n h_{n}^{d}\right)^{-2} \sum_{v_{n}<|i-j| \leq n} \alpha(|i-j|) \\
& \leq C_{6} n^{2}\left(n h_{n}^{d}\right)^{-2} \alpha\left(v_{n}\right) \\
& \leq C_{6} h_{n}^{d(v-2)}=\mathrm{o}(1)
\end{aligned}
$$

In conclusion, from (20) we have $V_{1} \longrightarrow 0$ and combining (23) and (24) in (22) we have $V_{2} \longrightarrow 0$ yields $\nu \longrightarrow 0$.

Proof of Theorem 2. According to the decomposition (16), we establish the next lemmas that estimates the negligible terms $\mathcal{B}_{1, \gamma}(x)$ and $\mathcal{B}_{3, \gamma}(x)$, the other lemmas control the main term $\mathcal{B}_{2, \gamma}(x)$, by calculating the variance and covariance.

Lemma 1. Under assumptions (H), (K)(ii) and (D)(i) we have for $\gamma=1,2$ and $n$ large enough

$$
\begin{aligned}
& \sqrt{n h_{n}^{d}}\left|\mathcal{B}_{1, \gamma}(x)\right|=o(1), \\
& \sqrt{n h_{n}^{d}}\left|\mathcal{B}_{3, \gamma}(x)\right|=o(1) .
\end{aligned}
$$

Proof of Lemma 1. Following the proof of lemmas 1 and 2 in [3], we get

$$
\begin{aligned}
& \sqrt{n h_{n}^{d}}\left|\mathcal{B}_{1, \gamma}(x)\right|=\mathrm{O}\left(\sqrt{h_{n}^{d} \log \log n}\right)=\mathrm{o}(1), \\
& \sqrt{n h_{n}^{d}}\left|\mathcal{B}_{3, \gamma}(x)\right|=\mathrm{O}\left(\sqrt{n h_{n}^{d+2}}\right)=o(1)
\end{aligned}
$$

which conclude the proof. 
Lemma 2. Under assumptions (K) and (D) with $\{\ell=2 \gamma$, for $\gamma=1,2\}$, we have

$$
n h_{n}^{d} \operatorname{Var}\left(\mathcal{B}_{2, \gamma}(x)\right) \longrightarrow \sigma_{\gamma}(x):=r_{\ell}(x) \int_{\mathbb{R}^{d}} K_{d}^{2}(t) d t
$$

Proof of Lemma 2. Let for $1 \leq i \leq n$

$$
U_{i, \gamma}(x):=\widetilde{T}_{i}^{-\gamma} K_{d}\left(\frac{x-X_{i}}{h_{n}}\right)-\mathbb{E}\left[\widetilde{T}_{i}^{-\gamma} K_{d}\left(\frac{x-X_{i}}{h_{n}}\right)\right], \quad \text { for } \gamma=1,2 .
$$

In terms of that quantity we have

$$
\mathcal{B}_{2, \gamma}(x)=\frac{1}{n h_{n}^{d}} \sum_{i=1}^{n} U_{i, \gamma}(x)
$$

For this, note that for $\gamma=1,2$

$$
\begin{aligned}
n h_{n}^{d} \operatorname{Var}\left(\mathcal{B}_{2, \gamma}(x)\right) & =\frac{1}{n h_{n}^{d}} \operatorname{Var}\left(\sum_{i=1}^{n} U_{i, \gamma}(x)\right) \\
& =\frac{1}{h_{n}^{d}} \mathbb{E}\left[U_{1, \gamma}^{2}(x)\right]+\frac{1}{n h_{n}^{d}} \sum_{\substack{i, j=1 \\
i \neq j}}^{n} \mathbb{E}\left[U_{i, \gamma}(x) U_{j, \gamma}(x)\right] \\
& =: \mathcal{A}_{1}+\mathcal{A}_{2} .
\end{aligned}
$$

We shall first study $\mathcal{A}_{1}$ in greater detail. We have

$$
\begin{aligned}
\mathcal{A}_{1} & =\frac{1}{h_{n}^{d}}\left\{\mathbb{E}\left[\frac{\delta_{1} Y^{-\ell}}{\bar{G}^{2}\left(Y_{1}\right)} K_{d}^{2}\left(\frac{x-X_{1}}{h_{n}}\right)\right]-\mathbb{E}^{2}\left[\widetilde{T}_{1}^{-\gamma} K_{d}\left(\frac{x-X_{1}}{h_{n}}\right)\right]\right\} \\
& =: \frac{1}{h_{n}^{d}}\left\{\mathcal{A}_{1,1}-\mathcal{A}_{1,2}\right\} .
\end{aligned}
$$

As a consequence of (18), for $\ell=2 \gamma$ with $\gamma=1$, 2, we get that

$$
\mathcal{A}_{1,1}=h_{n}^{d} r_{\ell}(x) \int K_{d}^{2}(t) d t
$$

and from (19) we obtain

$$
\mathcal{A}_{1,2} \leq \mathrm{O}\left(h_{n}^{2 d}\right) .
$$

Then, we join the two results obtained just before by

$$
\begin{aligned}
\mathcal{A}_{1} & \leq h_{n}^{-d}\left\{\mathcal{A}_{1,1}-\mathcal{A}_{1,2}\right\} \\
& \leq h_{n}^{-d}\left\{h_{n}^{d} r_{\ell}(x) \int K_{d}^{2}(t) d t-h_{n}^{2 d}\right\} \\
& =r_{\ell}(x) \int K_{d}^{2}(t) d t .
\end{aligned}
$$

From (21) we have

$$
\left|\mathbb{E}\left[U_{i, \gamma}(x) U_{j, \gamma}(x)\right]\right| \leq C h_{n}^{2 d} .
$$

Now, to deal with the term $\mathcal{A}_{2}$ we consider a sequence of integer $v_{n}$ defined before, so that

$$
\mathcal{A}_{2}=\frac{1}{n h_{n}^{d}}\left\{\sum_{1 \leq|i-j| \leq v_{n}} \mathbb{E}\left[U_{i, \gamma}(x) U_{j, \gamma}(x)\right]+\sum_{v_{n}<|i-j| \leq n} \mathbb{E}\left[U_{i, \gamma}(x) U_{j, \gamma}(x)\right]\right\} .
$$

On the one hand, from (26) we have

$$
\sum_{1 \leq|i-j| \leq v_{n}}\left|\mathbb{E}\left[U_{i, \gamma}(x) U_{j, \gamma}(x)\right]\right| \leq C h_{n}^{2 d} n v_{n}
$$


By choosing for $b>1, v_{n}=\left[\left(h_{n}^{-d}\right)^{1 / b}\right]$, we get

$$
\frac{1}{n h_{n}^{d}} \sum_{1 \leq|i-j| \leq v_{n}}\left|\mathbb{E}\left[U_{i, \gamma}(x) U_{j, \gamma}(x)\right]\right| \leq C h_{n}^{(b-1) d / b} \longrightarrow 0 .
$$

On the other hand, we use a version of the moment inequality due to [24]. Let $a_{1}, a_{2}$ and $a_{3}$ taking values in $\mathbb{R}^{\star}$ and greater or equal to 1 such that: $\frac{1}{a_{1}}+\frac{1}{a_{2}}+\frac{1}{a_{3}}=1$ and $b<a_{1}<\frac{v}{2}$. We have

$$
\begin{array}{r}
\frac{1}{n h_{n}^{d}} \sum_{v_{n}<|i-j| \leq n}\left|\mathbb{E}\left[U_{i, \gamma}(x) U_{j, \gamma}(x)\right]\right| \leq\left(n h_{n}^{d}\right)^{-1} \sum_{v_{n}<|i-j| \leq n} C \alpha^{\frac{1}{a_{1}}}(|i-j|) \\
\times\left(\mathbb{E}\left|K_{d}\left(\frac{x-X_{i}}{h_{n}}\right)\right|^{a_{2}}\right)^{\frac{1}{a_{2}}}\left(\mathbb{E}\left|K_{d}\left(\frac{x-X_{j}}{h_{n}}\right)\right|^{a_{3}}\right)^{\frac{1}{a_{3}}} .
\end{array}
$$

We have

$$
\mathbb{E}\left|K_{d}\left(\frac{x-X_{i}}{h_{n}}\right)\right|^{a_{2}} \leq C h_{n}^{d} .
$$

So, under (M)(ii) and for $b<a<\frac{v}{2}$ we have

$$
\begin{aligned}
\frac{1}{n h_{n}^{d}} \sum_{v_{n}<|i-j| \leq n} \mathbb{E}\left[U_{i, \gamma}(x) U_{j, \gamma}(x)\right] & \leq \frac{C}{n h_{n}^{d}} \sum_{v_{n}<|i-j| \leq n} \alpha^{\frac{1}{a_{1}}}(|i-j|)\left(h_{n}^{d}\right)^{\frac{1}{a_{2}}}\left(h_{n}^{d}\right)^{\frac{1}{a_{3}}} \\
& \leq \frac{C}{n h_{n}^{d}} \sum_{v_{n}<|i-j| \leq n} \alpha^{\frac{1}{a_{1}}}(|i-j|) h_{n}^{\frac{d}{a_{1}}\left(a_{1}-1\right)} \\
& \leq \frac{C}{n h_{n}^{\frac{d}{a_{1}}}} \sum_{v_{n}<i-j \mid \leq n} \alpha^{\frac{1}{a_{1}}}(|i-j|) \\
& \leq \frac{C}{n h_{n}^{\frac{d}{a_{1}}}} \sum_{v_{n}<|i-j| \leq n} \frac{|i-j|}{v_{n}} \alpha^{\frac{1}{a_{1}}}(|i-j|) \\
& \leq \frac{C}{n h_{n}^{\frac{d}{a_{1}}}}+\sum_{s>v_{n}} \frac{s}{h_{n}^{-\frac{d}{b}}} \alpha^{\frac{1}{a_{1}}}(s) \\
& \leq \frac{C}{n h_{n}^{d\left(\frac{1}{a_{1}}-\frac{1}{b}\right)}} \sum_{s>v_{n}} s \alpha^{\frac{1}{a_{1}}}(s) \longrightarrow 0 .
\end{aligned}
$$

Finally combining (27) and (28) we get the result of Lemma 2.

Lemma 3. Under assumptions (K) and (D)(i, iii), we have

$$
\operatorname{Cov}\left(\sqrt{n h_{n}^{d}} \mathcal{B}_{2,1}(x), \sqrt{n h_{n}^{d}} \mathcal{B}_{2,2}(x)\right) \longrightarrow \sigma_{3}(x):=r_{3}(x) \int_{\mathbb{R}^{d}} K_{d}^{2}(t) d t .
$$

Proof of Lemma 3. By definition we get

$$
\begin{aligned}
\sqrt{n h_{n}^{d}} \operatorname{Cov}\left(\mathcal{B}_{2,1}(x), \mathcal{B}_{2,2}(x)\right)= & n h_{n}^{d}\left\{\mathbb{E}\left[\mathcal{B}_{2,1}(x) \mathcal{B}_{2,2}(x)\right]-\mathbb{E}\left[\mathcal{B}_{2,1}(x)\right] \mathbb{E}\left[\mathcal{B}_{2,2}(x)\right]\right\} \\
=: & n h_{n}^{d}\left\{\mathcal{S}_{3}-\mathcal{S}_{1} \mathcal{S}_{2}\right\}
\end{aligned}
$$

On the one hand, we have

$$
\begin{aligned}
\mathcal{S}_{3} & =\mathbb{E}\left[\widetilde{\mu}_{1}(x) \widetilde{\mu}_{2}(x)\right]-\mathbb{E}\left[\widetilde{\mu}_{1}(x)\right] \mathbb{E}\left[\widetilde{\mu}_{2}(x)\right] \\
& =: \quad \mathcal{S}_{3,1}-\mathcal{S}_{3,2} .
\end{aligned}
$$


For $\mathcal{S}_{3,1}$ we have

$$
\begin{aligned}
\mathcal{S}_{3,1} & =\frac{1}{\left(n h_{n}^{d}\right)^{2}} \sum_{i, j=1} \mathbb{E}\left[\widetilde{T}_{i}^{-1} \widetilde{T}_{j}^{-2} K_{d}\left(\frac{x-X_{i}}{h_{n}}\right) K_{d}\left(\frac{x-X_{j}}{h_{n}}\right)\right] \\
& =\frac{1}{\left(n h_{n}^{d}\right)^{2}}\left\{\sum_{i, j=1}^{n} \mathbb{E}\left[\frac{\delta_{i} Y_{i}^{-3}}{\bar{G}^{2}\left(Y_{i}\right)} K_{d}^{2}\left(\frac{x-X_{i}}{h_{n}}\right)\right]\right. \\
& \left.+\sum_{\substack{i, j=1 \\
i \neq j}}^{n} \mathbb{E}\left[\widetilde{T}_{i}^{-1} \widetilde{T}_{j}^{-2} K_{d}\left(\frac{x-X_{i}}{h_{n}}\right) K_{d}\left(\frac{x-X_{j}}{h_{n}}\right)\right]\right\} \\
& =\frac{1}{\left(n h_{n}^{d}\right)^{2}}\left\{\sum_{i, j=1}^{n} \mathcal{S}_{3,1,1}+\sum_{\substack{i, j=1 \\
i \neq j}}^{n} \mathcal{S}_{3,1,2}\right\} .
\end{aligned}
$$

For $\mathcal{S}_{3,1,1}$, using the conditional expectation property, a change of variable and Taylor expansion in (17) for $\ell=3$, we have

$$
\begin{aligned}
\mathcal{S}_{3,1,1} & =\int K_{d}^{2}\left(\frac{x-u}{h_{n}}\right) \int \frac{t^{-3}}{\bar{G}(t)} f(t \mid u) d t f(u) d u \\
& =\int K_{d}^{2}\left(\frac{x-u}{h_{n}}\right) r_{3}(u) d u \\
& =h_{n}^{d} \int K_{d}^{2}(t) r_{3}\left(x-h_{n} t\right) d t \\
& \leq h_{n}^{d} r_{3}(x) \int_{\mathbb{R}^{d}} K_{d}^{2}(t) d t .
\end{aligned}
$$

From (26), we have $\left|\mathbb{E}\left[U_{i, \gamma}(x) U_{j, \gamma}(x)\right]\right| \leq C h_{n}^{2 d}$ then

$$
\begin{aligned}
\mathcal{S}_{3,1} & \leq\left(n h_{n}^{d}\right)^{-2}\left\{n h_{n}^{d} r_{3}(x) \int_{\mathbb{R}^{d}} K_{d}^{2}(t) d t+O\left(\left(n h_{n}^{d}\right)^{2}\right)\right\} \\
& =\left(n h_{n}^{d}\right)^{-1} r_{3}(x) \int_{\mathbb{R}^{d}} K_{d}^{2}(t) d t+o(1)
\end{aligned}
$$

which conclude the proof of Lemma 3.

In order to prove the asymptotic normality of $\sqrt{n h_{n}^{d}} \mathcal{B}_{2, \gamma}(x)$ for $\gamma=1,2$, we use the Doob technique (see : [8], pages 228 to 232). The idea is to split the $n$-sample into large and small blocks : $p$-blocks and $q$-blocks respectively. For this purpose, let $p:=p_{n}<n$ and $q:=q_{n}<n$ satisfying the assumption (M).

For a given pair of real numbers $\left(c_{1}, c_{2}\right)$, define

$$
\mathcal{B}_{2, n}(x):=\sqrt{n h_{n}^{d}} \sum_{\gamma=1}^{2} c_{\gamma} \mathcal{B}_{2, \gamma}(x)=c_{1} \sqrt{n h_{n}^{d}} \mathcal{B}_{2,1}(x)+c_{2} \sqrt{n h_{n}^{d}} \mathcal{B}_{2,2}(x)
$$

Lemma 4. Under assumptions (K), (B), (D) and (M) we have

$$
\mathcal{B}_{2, n}(x) \stackrel{\mathcal{D}}{\longrightarrow} \mathcal{N}\left(0, \sigma_{4}^{2}(x)\right)
$$

where $\sigma^{2}(x)=\kappa c^{t} \Sigma_{x} c$ for $c=\left(c_{1}, c_{2}\right)^{t}$ and

$$
\Sigma_{x}=\left(\begin{array}{ll}
r_{2}(x) & r_{3}(x) \\
r_{3}(x) & r_{4}(x)
\end{array}\right)
$$


Proof of Lemma 4. We have from (28) that

$$
\begin{aligned}
\operatorname{Var}\left(\mathcal{B}_{2, n}(x)\right) & =\operatorname{Var}\left(c_{1} \sqrt{n h_{n}^{d}} \mathcal{B}_{1,2}(x)+c_{2} \sqrt{n h_{n}^{d}} \mathcal{B}_{2,2}(x)\right) \\
& =c_{1}^{2} n h_{n}^{d} \operatorname{Var}\left(\mathcal{B}_{1,2}(x)\right)+c_{2}^{2} n h_{n}^{d} \operatorname{Var}\left(\mathcal{B}_{2,2}(x)\right)+2 c_{1} c_{2} n h_{n}^{d} \operatorname{Cov}\left(\mathcal{B}_{1,2}(x), \mathcal{B}_{2,2}(x)\right)
\end{aligned}
$$

which the variance terms have been studied in Lemma 2 and the covariance term has been treated in Lemma 3. Now let $W_{i}(x):=c_{1} U_{i, 1}(x)+c_{2} U_{i, 2}(x)$, then

$$
\mathcal{B}_{2, n}(x)=\frac{1}{\sqrt{n}} \sum_{i=1}^{n} \frac{W_{i}(x)}{\sqrt{h_{n}^{d}}}=: \frac{\widetilde{W}_{n}(x)}{\sqrt{n}} .
$$

Our main aim is to show that :

$$
\frac{\widetilde{W}_{n}(x)}{\sqrt{n}} \stackrel{\mathcal{D}}{\longrightarrow} \mathcal{N}\left(0, \sigma_{4}^{2}(x)\right)
$$

Using Doob's technique, under (B) we divide the set into $k$ large $p$-blocks and $k$ small $q$-blocks. Let

$$
\begin{aligned}
w_{1, j} & =\sum_{i=(j-1)\left(p_{n}+q_{n}\right)+1}^{j\left(p_{n}+q_{n}\right)+p_{n}} \frac{W_{i}(x)}{\sqrt{h_{n}^{d}}}, \\
w_{2, j} & =\sum_{i=(j-1)\left(p_{n}+q_{n}\right)+p_{n}+1}^{j\left(p_{n}+q_{n}\right)} \frac{W_{i}(x)}{\sqrt{h_{n}^{d}}}, \\
w_{3, k} & =\sum_{i=k\left(p_{n}+q_{n}\right)+1}^{n} \frac{W_{i}(x)}{\sqrt{h_{n}^{d}}}
\end{aligned}
$$

and

$$
W_{1, n}=\sum_{j=1}^{k} w_{1, j}, \quad W_{2, n}=\sum_{j=1}^{k} w_{2, j} \quad \text { and } \quad W_{3, n}=\sum_{j=1}^{k} w_{3, j}
$$

where $W_{n}(x)=W_{1, n}+W_{2, n}+W_{3, n}$.

$$
\frac{1}{\sqrt{n}} W_{1, n} \stackrel{\mathcal{D}}{\longrightarrow} \mathcal{N}\left(0, \sigma_{4}^{2}(x)\right),
$$

and

$$
\frac{1}{n} \mathbb{E}\left[W_{2, n}^{2}\right]+\frac{1}{n} \mathbb{E}\left[W_{3, n}^{2}\right] \longrightarrow 0 .
$$

We start by proving (31)

$$
\begin{aligned}
\mathbb{E}\left[W_{2, n}^{2}\right] & =\mathbb{E}\left[\left(\sum_{j=1}^{k} w_{2, j}\right)^{2}\right] \\
& =\sum_{j=1}^{k} \operatorname{Var}\left(w_{2, j}\right)+2 \sum_{1 \leq j, l \leq k} \operatorname{Cov}\left(w_{2, j}, w_{2, \ell}\right) .
\end{aligned}
$$

On the one hand, we have

$$
\begin{aligned}
\operatorname{Var}\left(w_{2, j}\right) & =\operatorname{Var}\left(\sum_{i=(j-1)\left(p_{n}+q_{n}\right)+p_{n}+1}^{j\left(p_{n}+q_{n}\right)} \frac{W_{i}(x)}{\sqrt{h_{n}^{d}}}\right) \\
& =q_{n} \operatorname{Var}\left(\frac{W_{1}(x)}{h_{n}^{d}}\right)+\sum_{0 \leq|i-j| \leq q_{n}} \operatorname{Cov}\left(\frac{W_{i}(x)}{h_{n}^{d}}, \frac{W_{l}(x)}{h_{n}^{d}}\right) \\
& \leq q_{n} \operatorname{Var}\left(\frac{W_{1}(x)}{\sqrt{h_{n}^{d}}}\right)+\mathrm{o}\left(q_{n}\right) .
\end{aligned}
$$


So,

$$
\sum_{j=1}^{k} \operatorname{Var}\left(w_{2, j}\right)=k q \operatorname{Var}\left(\frac{W_{1}(x)}{\sqrt{h_{n}^{d}}}\right)+o\left(k q_{n}\right) .
$$

On the other hand, we have

$$
\operatorname{Var}\left(\frac{W_{1}(x)}{\sqrt{h_{n}^{d}}}\right)=c_{1}^{2} \sigma_{1}^{2}(x)+c_{2}^{2} \sigma_{2}^{2}(x)+2 c_{1} c_{2} \sigma_{3}(x)+\mathrm{o}(1) .
$$

From assumption (B) we have that $k q_{n}=\frac{n q_{n}}{\left(p_{n}+q_{n}\right)}=\mathrm{o}(n)$ then

$$
\sum_{j=1}^{k} \operatorname{Var}\left(w_{2, j}\right)=o(n) .
$$

Now, by stationary and assumption (M)(ii), we have

$$
\begin{aligned}
2 \sum_{j, \ell=1}^{k} \operatorname{Cov}\left(w_{2, j}, w_{2, \ell}\right) & \leq C \frac{k q_{n}}{h_{n}^{d}} \sum_{l=1}^{k-1} \sum_{m=1}^{q_{n}} h^{d\left(1-\frac{1}{a_{1}}\right)} \alpha^{\frac{1}{a_{1}}}\left(l\left(p_{n}+q_{n}\right)+m\right) \\
& \leq C \frac{k q_{n}}{h^{\frac{d}{a}}} \sum_{j \geq p_{n}+q_{n}+1} \alpha^{\frac{1}{a_{1}}}(j)=\mathrm{o}(n) .
\end{aligned}
$$

Then, from (32), (36) and (37), we deduce that

$$
\frac{1}{n} \mathbb{E}\left[W_{2, n}^{2}\right] \underset{n \rightarrow \infty}{\longrightarrow} 0 .
$$

Now, we treat the term $W_{3, n}$ in the same spirit of $W_{2, n}$, we have

$$
\begin{aligned}
\frac{1}{n} \mathbb{E}\left[W_{3, n}^{2}\right] & =\frac{1}{n}\left\{\left(n-k\left(p_{n}+q_{n}\right)\right) \operatorname{Var}\left(\frac{W_{1}(x)}{\sqrt{h_{n}^{d}}}\right)\right. \\
& \left.+\sum_{0 \leq|i-j| \leq n-k\left(p_{n}+q_{n}\right)} \operatorname{Cov}\left(\frac{W_{i}(x)}{\sqrt{h_{n}^{d}}}, \frac{W_{j}(x)}{\sqrt{h_{n}^{d}}}\right)\right\} \\
& \leq\left(1-\frac{k\left(p_{n}+q_{n}\right)}{n}\right) \operatorname{Var}\left(\frac{W_{1}(x)}{\sqrt{h_{n}^{d}}}\right)+\mathrm{o}(1) .
\end{aligned}
$$

Under assumption (B) we know that $\frac{k\left(p_{n}+q_{n}\right)}{n} \rightarrow 1$, then we get

$$
\frac{1}{n} \mathbb{E}\left[W_{3, n}^{2}\right] \longrightarrow 0 \text {. }
$$

So, (38) and (39) conclude the proof of (31).

Now concerning (30), let $\psi(s)$ and $\psi(j)$ be the characteristics function of $\frac{1}{\sqrt{n}} W_{1, n}$ and $w_{1, j}$ respectively. We show that

$$
\lim \left|\psi(x)-\prod_{j=1}^{k} \psi_{j}(s)\right|=0
$$

which proves that the r.v.'s $w_{1, j}$ are asymptotically independent, and then, prove that

$$
\prod_{j=1}^{k} \psi_{j}(s) \stackrel{\mathcal{D}}{\longrightarrow} \mathcal{N}\left(0, \sigma_{4}^{2}(x)\right) .
$$

For the first part, we have

$$
\begin{aligned}
\left|\psi(s)-\prod_{j=1}^{k} \psi_{j}(s)\right| & =\mathbb{E}\left[e^{i t \frac{W_{1, n}}{\sqrt{n}}}\right]-\prod_{j=1}^{k} \mathbb{E}\left[e^{i t w_{1, j}}\right] \\
& \leq 16 k \alpha\left(q_{n}+1\right) \leq C k \alpha\left(q_{n}\right) \longrightarrow 0 .
\end{aligned}
$$


For the second part, we have

$$
\frac{1}{n} \sum_{j=1}^{k} \mathbb{E}\left[w_{1, j}^{2}\right] \longrightarrow \sigma_{4}^{2}(x)
$$

and for all $\epsilon>0$

$$
\frac{1}{n} \sum_{j=1}^{k} \mathbb{E}\left[w_{1, j}^{2} \mathbb{1}_{\left\{w_{1, j}>\epsilon \sqrt{n} \sigma_{4, n}(x)\right\}}\right] \longrightarrow 0 .
$$

Furthermore

$$
\begin{aligned}
\operatorname{Var}\left(w_{1, j}\right) & =\left\{p_{n} \operatorname{Var}\left(\frac{W_{1}(x)}{\sqrt{h_{n}^{d}}}\right)+\sum \sum_{0<|i-\ell| \leq p_{n}} \operatorname{Cov}\left(\frac{W_{i}(x)}{\sqrt{h_{n}^{d}}}, \frac{W_{\ell}(x)}{\sqrt{h_{n}^{d}}}\right)\right\} \\
& \leq p_{n} \operatorname{Var}\left(\frac{W_{1}(x)}{\sqrt{h_{n}^{d}}}\right)+\mathrm{o}\left(p_{n}\right) .
\end{aligned}
$$

So

$$
\frac{1}{n} \sum_{j=1}^{k} \mathbb{E}\left[w_{1, j}^{2}\right]=\frac{k p_{n}}{n} \operatorname{Var}\left(\frac{W_{1}(x)}{\sqrt{h_{n}^{d}}}\right)+o\left(\frac{k p_{n}}{n}\right) .
$$

From (35) and knowing that $\frac{p_{n} k}{n} \rightarrow 1$ (40) is proved.

Now, to establish (41) we have that $\frac{W_{i}(x)}{\sqrt{h_{n}^{d}}} \leq \frac{C}{\sqrt{h_{n}^{d}}}$. Then under (B)

$$
\frac{1}{\sqrt{h_{n}^{d}}}\left|w_{1, j}\right| \leq \frac{C p_{n}}{\sqrt{n h_{n}^{d}}} \rightarrow 0 .
$$

Then the set $\left\{\left|w_{1, j}\right|>\epsilon \sqrt{n} \sigma_{4, n}(x)\right\}$ is empty for $n$ large enough and conclude the proof of (41).

Corollary 2. Under assumptions (K) and (D), for $\gamma=1$, 2, we have

$$
\sqrt{n h_{n}^{d}} \mathcal{B}_{2, \gamma}(x) \stackrel{\mathcal{D}}{\longrightarrow} \mathcal{N}\left(0, \sigma_{\gamma}^{2}(x)\right) .
$$

Proof of Corollary 2.If $\gamma=1$ and we take $c_{1}=1$ and $c_{2}=0$ (If $\gamma=2$ and we take $c_{1}=0$ and $c_{2}=1$ respectively) in (28), using the results in Lemma 1 and Lemma 4 we get the result.

Corollary 3. Under assumptions $(K)$ and $(D)$, we have

$$
\sqrt{n h_{n}^{d}}\left(\mathcal{B}_{2,1}(x), \mathcal{B}_{2,2}(x)\right) \stackrel{\mathcal{D}}{\longrightarrow} \mathcal{N}\left(0, \Sigma_{x} \int_{\mathbb{R}^{d}} K_{d}^{2}(t) d t\right)
$$

where $\Sigma_{x}$ is defined in Lemma 4.

Proof of Corollary 3. Using the results in Lemma 4 and Corollary 2 we get the result.

Corollary 4. Define the mapping $\zeta: \mathbb{R}^{2} \longrightarrow \mathbb{R}$ such that $\zeta\left(x_{1}, x_{2}\right)=\frac{x_{1}}{x_{2}}$ with $x_{2} \neq 0$, then

$$
\begin{aligned}
\widehat{m}(x)-m(x) & =\frac{\widehat{\mu}_{1}(x)}{\widehat{\mu}_{2}(x)}-\frac{\mu_{1}(x)}{\mu_{2}(x)} \\
& =\zeta\left(\widehat{\mu}_{1}(x), \widehat{\mu}_{2}(x)\right)-\zeta\left(\mu_{1}(x), \mu_{2}(x)\right) .
\end{aligned}
$$

By using the delta method we get

$$
\sqrt{n h_{n}^{d}}(\widehat{m}(x)-m(x)) \stackrel{\mathcal{D}}{\longrightarrow} \mathcal{N}\left(0, \nabla \zeta^{t} \Sigma_{x} \nabla \zeta \int_{\mathbb{R}^{d}} K_{d}^{2}(t) d t\right)
$$

where $\nabla \zeta^{t}=\left(\frac{\partial \zeta}{\partial x_{1}}, \frac{\partial \zeta}{\partial x_{2}}\right)$ is the gradient at point $\left(\mu_{1}(x), \mu_{2}(x)\right)$. So an elementary calculus gives $\sigma^{2}(x)$ defined in Theorem 2. 
Acknowledgments: The authors are grateful to the Editor and two anonymous reviewers whose careful reading enabled us to improve the quality of the paper and to enrich the simulations part with other models, confidence intervals and by adding the real data example.

Conflict of interest statement: Authors state no conflict of interest.

\section{References}

[1] Andersen, P. K., O. Borgan, R. D. Gill, and N. Keiding (1993). Statistical Models Based on Counting Processes. Springer, New York.

[2] Bosq, D. (1998). Nonparametric Statistics for Stochastics Processes. Estimation and Prediction. Springer, New-York.

[3] Bouhadjera, F. and E. Ould Said (2019). On the strong uniform consistency for relative error of the regression function estimator for censoring times series model. Available at https://arxiv.org/abs/1910.01964.

[4] Cai, J. and R. Prentice (1995). Estimating equations for hazard ratio parameters based on correlated failure time data. Biometrika 82(1), 151-164.

[5] Cai, Z. (1998). Asymptotic properties of Kaplan-Meier estimator for censored dependent data. Statist. Probab. Lett. 37(4), 381-389.

[6] Cai, Z. (2001). Estimating a distribution function for censored time series data. J. Multivariate Anal. 78(2), 299-318.

[7] Doukhan, P, Massart, P. and R. Rio, (1994). The functional central limit theorem for strongly mixing processes. Ann. Inst. Henri Poincaré: Probab. Statist. 30(1), 63-82.

[8] Doob, J. L. (1953). Stochastic Processes. Wiley, New York.

[9] El Ghouch, A. and I. Van Keilegom (2008). Nonparametric regression with dependent censored data. Scand. J. Stat. 35(2), 228-247.

[10] El Ghouch, A. and I. Van Keilegom (2009). Local linear quantile regression with dependent censored data. Statist. Sinica 19, $1621-1640$

[11] Fan, J. and I. Gijbels (1996). Local Polynomial Modelling and its Applications. Chapman \& Hall/CRC, Boca Raton FL.

[12] Fan, J. and Q. Yao (2003). Nonlinear Time Series: Nonparametric and Parametric Methods. Springer, New York.

[13] Guessoum, Z. and E. Ould Saïd (2008). On nonparametric estimation of the regression function under random censorship model. Statist. Decisions 26, 159-177.

[14] Guessoum, Z. and E. Ould Saïd (2010). Kernel regression uniform rate estimation for censored data under alpha-mixing condition. Electron. J. Statist. 4, 117-132.

[15] Guessoum, Z. and E. Ould Saïd (2012). Central limit theorem for the kernel estimator of the regression function for censored time series. J. Nonparametr. Stat. 24, 379-397.

[16] Hu, D. H. (2019). Local least product relative error estimation for varying coefficient multiplicative regression model. Acta. Math. Appl. Sinica 35, 274-286.

[17] Jones, D. A. (1978). Nonlinear autoregressive processes. Proc. R. Soc. London A 360, 71-95.

[18] Jones, M. C., H. Park, K. I. Shin, S. K. Vines, and S. O. Jeong (2008). Relative error prediction via kernel regression smoothers. J. Statist. Plann. Inference 138(10), 2887-2898.

[19] Kaplan, E. L. and P. Meier (1958). Nonparametric estimation from incomplete observations. J. Amer. Statist. Assoc. 53(282), 458-481.

[20] Khardani, S. and Y. Slaoui (2019). Nonparametric relative regression under random censorship model. Statist. Probab. Lett. 151, 116-122.

[21] Lipshitz, S. R. and J. G. Ibrahim (2000). Estimation with correlated censored survival data with missing covariates. Biostatistics 1(3), 315-327.

[22] Klein, J. P. and M. L. Moeschberger (2004). Survival Analysis: Techniques for Censored and Truncated Data. Springer, New York.

[23] Park, H. and L. A. Stefanski (1998). Relative error prediction. Statist. Probab. Lett. 40(3), 227-236.

[24] Rio, E. (2000). Theorie Asymptotique des Processus Aléatoires Faiblement Dépendants. Springer, Berlin.

[25] Rosenblatt, M. (1956). Remark on some nonparametric estimates of density function. Ann. Math. Statist. 27(3), $832-837$.

[26] Silverman, B. W. (1986). Density Estimation for Statistics and Data Analysis. Chapman and Hall, London.

[27] Venables, W. and B. Ripley (2002a). Modern Applied Statistics with S. Springer, New York.

[28] Venables, W. N. and B. D. Ripley (2002b). Modern Applied Statistics with S. Fourth edition. Springer, New York.

[29] Wei, L., Lin, D. and L. Weissfeld (1989). Regression analysis of multivariate incomplete failure time data by modeling marginal distributions. J. Amer. Statist. Assoc. 84(408), 1065-1073. 